\title{
Cation Hydration Energy and Surface Hydration Have Crucial Role On Thermodynamics of Clay
}

\section{Swelling}

\author{
Sai Adapa and Ateeque Malani*
}

Department of Chemical Engineering, Indian Institute of Technology Bombay, Mumbai 400076, India

E-mail: amalani@iitb.ac.in

Phone: +91-22-25767205. Fax: +91-22-2572 6895

\section{TOC Graphics:}

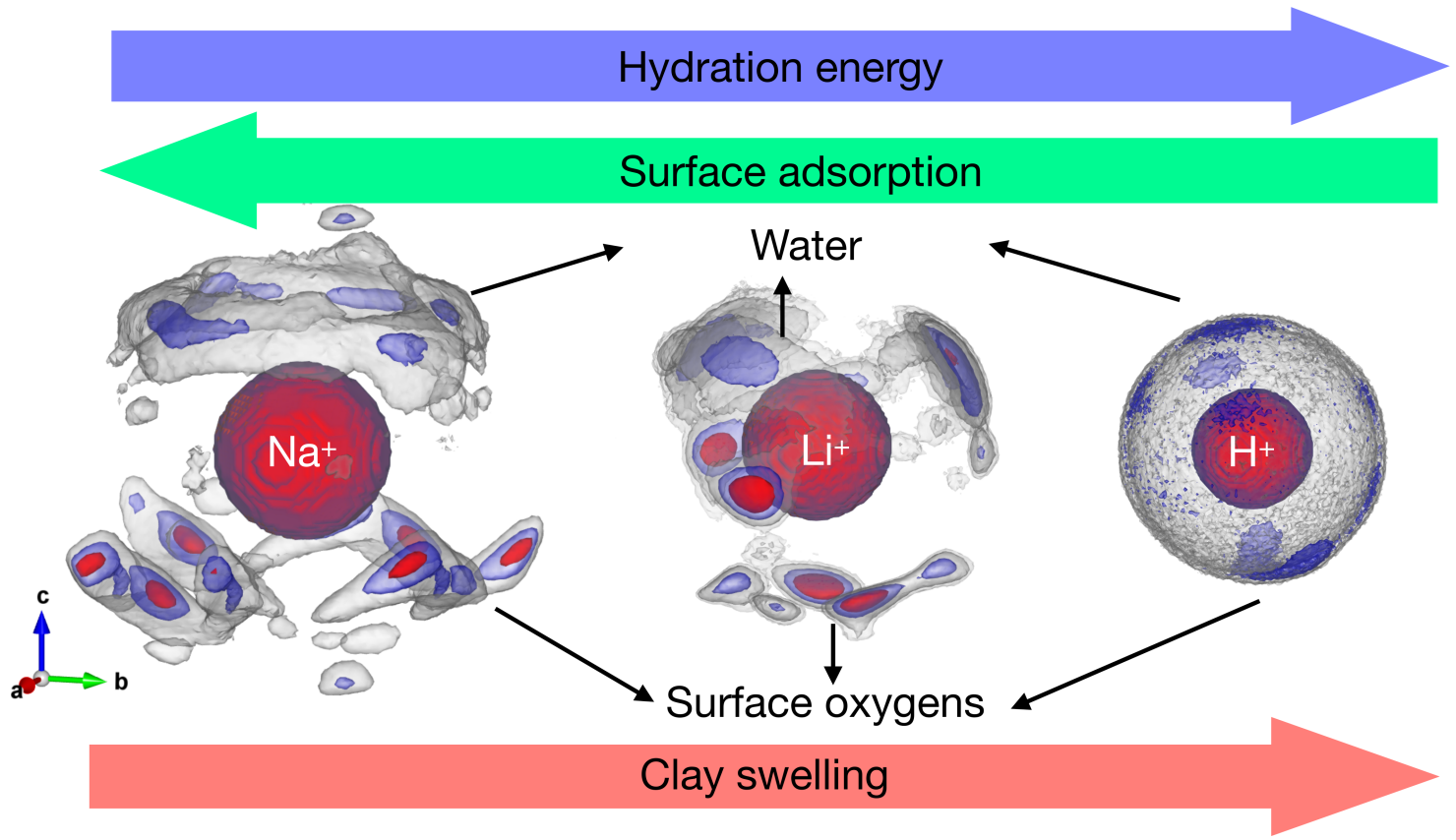




\begin{abstract}
The swelling capacity and stability of clay plays a crucial role in various areas ranging from cosmetics to oil extraction; hence change in their swelling behaviour after cation exchange with surrounding medium is important for their efficient utilisation. Here we focus on understanding the role of different hydration properties of cation on thermodynamics of clay swelling by water adsorption. We have used mica as the reference clay, $\mathrm{Na}^{+}, \mathrm{Li}^{+}$, and $\mathrm{H}^{+}$ions as the interstitial cations, and performed grand canonical Monte Carlo simulations of water adsorption in mica pores (of widths $d=$ 4-40 $\AA$ ). We found that water adsorption in Na-, Li- and H-mica pores is qualitatively similar; however significant quantitative differences are observed, specially at smaller d. Higher water density in H-mica pores $\left(\rho_{H}\right)$ was expected due smaller size of $\mathrm{H}^{+}$ions having higher hydration energy. However the counter-intuitive trend of $\rho_{L i}>\rho_{N a}>\rho_{b}$ (bulk density) $>\rho_{H}$ was observed due to adsorption energy where contribution of mica framework atoms was also found to be significant. The disjoining pressure (П), swelling free energy $\left(\Delta \Omega^{e x}\right)$, and several structural properties of confined water and ions were calculated to perform thermodynamic analysis of the system. Our detailed calculations have captured the structural evolution of ions and water, especially the transitions from mono- to bi- and multilayer as a function of $d$. An oscillatory behaviour in the $\Pi$ and $\Delta \Omega^{e x}$ profiles with diminishing to zero for $d \geq 11 \AA$ is observed in all three mica system. A shift in location of global minima of $\Delta \Omega^{e x}$ towards the higher $d$ values and $\Delta \Omega^{e x}$ becoming more repulsive is observed in the increasing order of hydration energy of $\mathrm{Na}^{+}, \mathrm{Li}^{+}$, and $\mathrm{H}^{+}$ions. The $\Delta \Omega^{e x}$ for Na-mica is characterised by global minima at $d=6 \AA$ corresponding to crystalline swelling, significant barrier for crystalline swelling from $d=6$ to $9 \AA$ and lower for crystalline $(d=9 \AA)$ to osmotic swelling $(d>12 \AA)$. For Li-mica, the energy barrier for crystalline to osmotic swelling is lesser compared to Na-mica system, whereas for H-mica the $\Delta \Omega^{e x}>0$ for all $d$ favouring osmotic swelling. We found that hydration of cations by surface atoms play key role in thermodynamics of clay swelling. The $\mathrm{Na}^{+}$ions hydrate more number of surface oxygens, acts as anchor, and hold the mica pore at $d=6 \AA$ by sharing hydrating water with ions of opposite
\end{abstract}


side forming an electrostatically connected bridge of mica-Na-water-Na-mica. The $\mathrm{Li}^{+}$ ions do hydrate surface oxygen atoms, albeit lesser number and sharing of hydration shell with nearby $\mathrm{Li}^{+}$ions is also minimum. Both, surface hydration and water sharing, is minimum in $\mathrm{H}^{+}$ion case, as they are mostly present in the center of the pore as diffusive ions; thus exerting a consistent osmotic pressure on the mica frameworks, favouring swelling.

\section{Introduction}

Traditionally, clay swelling has been investigated due to its vital role in oil and gas extraction. During drilling and extraction of oil, generally water based fluid is used to lubricate the drill, carry rock cutting and displace oil. However, the drilling fluid intercalates nearby rock and clay leading to swelling, which may reduce oil production and collapse of well. To prevent these technical problems and design suitable inhibitors, understanding fundamentals of clay swelling is important. Recently, clay swelling has found a renewed interest due to wide reach of importance of clays in $\mathrm{CO}_{2}$ sequestration, ${ }^{1}$ pharmaceuticals, ${ }^{2}$ cosmetics,${ }^{3}$ disposal of radioactive waste, ${ }^{4,5}$ nutrient supply in soil, ${ }^{6}$ cement, ${ }^{7}$ paint,${ }^{8}$ catalysis ${ }^{9}$ and many other industries. Clays are layered materials made of tetrahedral and octahedral sheets with isomorphic substitution in these layers which create charge imbalance, compensated by the loose, often hydrated, interstitial cations. The composition of clays (both, framework atoms and cations) vary locally at platelets level (in $\mathrm{nm}$ to micron range) and globally (at cm to km range) due to geological process, geographical location and climate. Further, clays could have different interstitial cation due to ion exchange with the surrounding medium occurring at various time scales. Here we investigate the role of cation hydration properties (hydration energies, size, water and surface-hydration structure, and coordination number) on swelling pressure and free energy of the clays.

The ion exchange and swelling process would generally occur simultaneously in reality; however, capturing this complex process using current computational methods is a challenge. 
Hence the process is divided into two separate steps; i) ion exchange in presence of surrounding reservoir and ii) swelling assuming that ion-exchange is completed. Similar assumption has been used by several researchers and they probed ion exchange and swelling separately. For example, Rotenberg and coworkers ${ }^{10}$ has studied ion exchange free energy between interlayer zone and surrounding in montmorillonite (MMT) clay for a fixed interlayer spacing containing two water layers. They found that exchange of cations are not activated, however, insertion of anion in the interlayer zone is not favourable. After the cation exchange, the clay nanopore would re-adjust to equilibrium interlayer spacing by the exchange of water molecules with the reservoir, as recently shown in the experimental study. ${ }^{11}$ The stability of clays, their rheological properties, and, mobility of confined water and ions are significantly affected after clay swelling. ${ }^{12}$ Hence knowledge of clay swelling is important for its efficient utilisation in varied applications.

Early work of Hofmann et al. ${ }^{13}$ had established the swelling of MMT clays due to water adsorption in the interlayer spaces using X-ray diffraction (XRD) technique. Since then several studies have probed swelling of clays in presence of water vapors (at various relative humidities, RH), liquid water reservoir and electrolyte solution. ${ }^{11,14-36}$ Reviewing all of them is a challenge, hence, we will focus on few studies which probed swelling behaviour with different cations. Mooney et al. ${ }^{17}$ studied adsorption of water vapor at various $\mathrm{RH}$ in cation exchange MMT (M-MMT) and found that the amount of water adsorbed at $\mathrm{RH}=50 \%$ is highest in H-MMT ( $200 \mathrm{mg}$ of water/g of clay), followed by Li- and Na-MMT (145 - 135 $\mathrm{mg} / \mathrm{g})$ and smaller quanitities in $\mathrm{K}, \mathrm{Rb}$, and Cs-MMTs $(105-95 \mathrm{mg} / \mathrm{g})$. Berend et al. ${ }^{18}$ also studied water adsorption in MMT with several exposed cations and found one water layer (1WL) at lower $\mathrm{RH}$ and two water layers (2WLs) at higher $\mathrm{RH}$ in $\mathrm{Li}-, \mathrm{Na}-$, and K-MMT. The swelling behaviour of K-, Rb-, and Cs-MMT is similar and shows narrow hysteresis loop, whereas for Li- and Na-MMT, the change in basal spacing behaviour shows multiple jumps, highlighting the role of hydration energy of cations. ${ }^{18}$ Similar observation of $1 \mathrm{WL}$ and $2 \mathrm{WL}$ clays in Li-, Na-, and K-MMT has been observed in recent water vapor 
adsorption studies as well. ${ }^{19,20}$ Norrish $^{14}$ studied MMT swelling due to various ion and found both crystalline (interlayer spacing less than $20 \AA$ ) and osmotic swelling (continuous swelling beyond $20 \AA$ ) in presence of reservoir of both, pure water and electrolyte solution of corresponding cations. He found that monovalent cations of higher hydration energy exhibits higher swelling whereas monovalent ions of lower hydration energy and divalent ions with significantly higher hydration energy shows lesser swelling. He claimed that structural arrangement and interaction of confined water with oxygens of silica sheet of clay plays an important role in swelling of clays. He proposed that the free energy of the clay swelling should be a balance of a) energy required to separate the clay sheets and b) hydrtaion of ions; however, no explicit calculations were performed. Posner and Quirk ${ }^{21}$ also studied clay swelling in presence of electrolyte solutions of several mono and divalent cations using XRD. They also observed change in interlayer spacing from $2 \mathrm{WL}$ to $3 \mathrm{WL}$ for Li- and NaMMT. Further, a collapse of clays to lower interlayer spacing was observed with increase in concentration of electrolyte solution. Using the law of mass action, they calculated the free energy change for swelling corresponding to $1 \mathrm{WL}$ change (i.e., nWL $\rightarrow(\mathrm{n}+1) \mathrm{WL})$ and found to be directly related with the hydration energy of cation. In a recent study, the confined fluids were visualised using transmission electron microscopy and found that $\mathrm{K}^{+}$ions remain close to the surface of MMT, whereas $\mathrm{Na}^{+}$ions are located in the center of the interstitial zone also leading to more number of confined WLs. ${ }^{11}$

In the atomic force microscopy (AFM) and surface force apparatus (SFA) experiments, the forces exerted on the clay surfaces in presence of electrolyte solution is directly measured. At larger interlayer distances, the force profile is very well explained by the DLVO theory, ${ }^{34,37,38}$ whereas at smaller distances, the repulsive region is attributed to hydration forces. Several groups have performed AFM and SFA experiments of many cations at different electrolyte concentrations ${ }^{30,31,35,36,39-45}$ and the general observations are that at low concentration snap-in (joining of two surfaces) is observed, whereas repulsive hydration force is observed beyond a critical concentration of electrolyte. For example, Pashley ${ }^{34}$ observed 
the repulsive hydration forces beyond $4 \times 10^{-5}, 10^{-3}, 10^{-2}$, and $6 \times 10^{-2} M$ electrolyte concentration of $\mathrm{K}^{+}, \mathrm{Cs}^{+}, \mathrm{Na}^{+}$, and $\mathrm{Li}^{+}$cations, claimed to be associated with the size, hydration energy and surface concentration of adsorbed cations on clay surface. At higher electrolyte concentrations, oscillatory force profile with significant minimas and periodicity of $2.5 \AA$ is observed which was claimed to be due to dehydration of cations. ${ }^{35,39}$ In a recent AFM study, ${ }^{40}$ higher repulsive hydration strength and oscillation in force profile for $\mathrm{Li}^{+}$and $\mathrm{Na}^{+}$ cations as compared to $\mathrm{K}^{+}$and $\mathrm{Rb}^{+}$cations were observed. Further, the mechanical energy required to bring the AFM tip closer to the surface was found to be directly correlated with the hydration energy of the cations. ${ }^{40}$

Computational studies of clay swelling have generally used either Monte Carlo (MC) or molecular dynamics (MD) technique to probe equilibrium water loading, interstitial spacing, and structure and dynamics of confined water and ions. ${ }^{16,22,46-64}$ Here, we discuss those studies where different cations were probed to elucidate their role in clay swelling. Most of these simulations are performed at different water loading to obtain the equilibrium interlayer spacing and clay-hydration energies (energy change due to addition of water in dry clay) to comment on the swelling capacity of studied clays. For example, Boek et al. ${ }^{48}$ observed a significantly higher attractive clay-hydration energy for Na- and Li-MMTs whereas a repulsive behaviour was observed for K-MMT. Due to different hydration energies of the cations, they were found to be located either closer to the surface $\left(\mathrm{K}^{+}\right.$ions) or in the center of the pore ( $\mathrm{Li}^{+}$and $\mathrm{Na}^{+}$ions). Recently Teich-McGoldrick et al. ${ }^{55}$ performed serveral MD simulations and found that the clay-hydration energy exhibited a broad minima at 1WL for Na- and Cs-beidellite (BDL = MMT with framework charge located at silica tetrahedral sheet) whereas a steeper minima at $1 \mathrm{WL}$ is observed for $\mathrm{Mg}$ - and Ca-BDLs. At smaller interlayer spacing corresponding to $1 \mathrm{WL}$, the $\mathrm{Na}^{+}$and $\mathrm{Cs}^{+}$ions are located closer to the BDL surface and at higher basal spacing of $2 \mathrm{WL}$, an additional layer of the $\mathrm{Na}^{+}$ions at the center is observed, whereas $\mathrm{Cs}^{+}$ions are located closer to the BDL surface. Most of the studies have commented on swelling capacity of clays based on the clay-hydration energy, however, 
only few studies have calculated the complete thermodynamics of the system. Whitley and Smith ${ }^{58}$ performed MC simulations to obtain thermodynamics of swelling behaviour in Na-, Cs-, and Sr-MMT. They found that the grand potential (also referred here as swelling free energy) exhibits multiple minima with global minimum located at 1WL for Cs-MMT and 2WL for Na- and Sr-MMT indicating that hydration energy and valency has significant effect. The authors comments that pressure in the confined system was correlated with the hydration number of confined ions. Recently, Shen and Bourg ${ }^{63}$ have performed MD simulations to obtain free energy between two MMT colloidal particles at various $\mathrm{NaCl}$ salt concentration in presence of explicit water. In pure water case, they found the free energy minima at three locations with global minima located at $6 \AA$. The above studies reproduces the experimentally observed minima in free energy profile corresponding to 1,2 , and $3 \mathrm{WL}$ as well. At higher salt concentrations, they do not observe the free energy barrier between crystalline and osmotic swelling. While these studies investigated the structure of confined water and ions, clay-hydration energy in detail; however the individual pair interactions i.e., ion-surface, water-surface and surface-surface interactions have not been investigated in details. Recently, Shen and Bourg ${ }^{63}$ and Li et al. ${ }^{64}$ have probed these individual interactions and found a significant role in the swelling free energy of clays.

Table 1: Hydration properties of $\mathrm{Na}^{+}, \mathrm{Li}^{+}$, and $\mathrm{H}^{+}$ions in bulk-liquid water.

\begin{tabular}{lllll}
\hline \hline Ions & $\Delta G_{\text {hyd }}$ & $r_{\text {max }}$ & $r_{\min }$ & $C_{n}$ \\
\hline $\mathrm{Na}^{+}$ & -369.86 & 2.38 & 3.25 & 5.95 \\
$\mathrm{Li}^{+}$ & -474.04 & 2.0 & 2.80 & 4.49 \\
$\mathrm{H}^{+}$ & -1050.0 & 2.0 & 2.50 & 2.00 \\
\hline$\Delta G_{\text {hyd }}$ (kJ/mol) & - hydration energy of ion in bulk- \\
water from Ref. & $65,66, r_{\max }$ and $r_{\text {min }}(\AA)$ - location of \\
the first maximum and minimum of ion-water g(r), $C_{n}$ \\
- coordination number.
\end{tabular}

Our focus is to understand the role of cation size, their hydration energy on the thermodynamics of the clay swelling. We have chosen muscovite mica as the reference clay and considered three cations $-\mathrm{Na}^{+}, \mathrm{Li}^{+}$, and $\mathrm{H}^{+}$ions- as the interstitial ions. These ions were 
chosen because they have significant difference in hydration energy and coordination number due to differences in their sizes (see Table 1). We have performed grand canonical MC (GCMC) simulations of water adsorption from the implicit bulk-liquid reservoir at various interlayer spacing (pore width, $d$ ). From the trajectory of the simulations, we calculated disjoining pressure exerted on the mica framework - total as well as individual contribution from confined water, cations and opposite mica framework - from which swelling free energy (total and individual contribution) was evaluated. We found significant differences in swelling free energy which was strongly related to the structure and hydration status of confined ions. We explained these observations from the analaysis of ion-hydration (due to surface and water), sharing of hydration shells of ions by water molecules, density profiles of water and ions, and orientational analysis of water molecules. In the next section we describe the simulation methodology in detail, followed by results and discussions and finally we provide the summary and few conclusions.

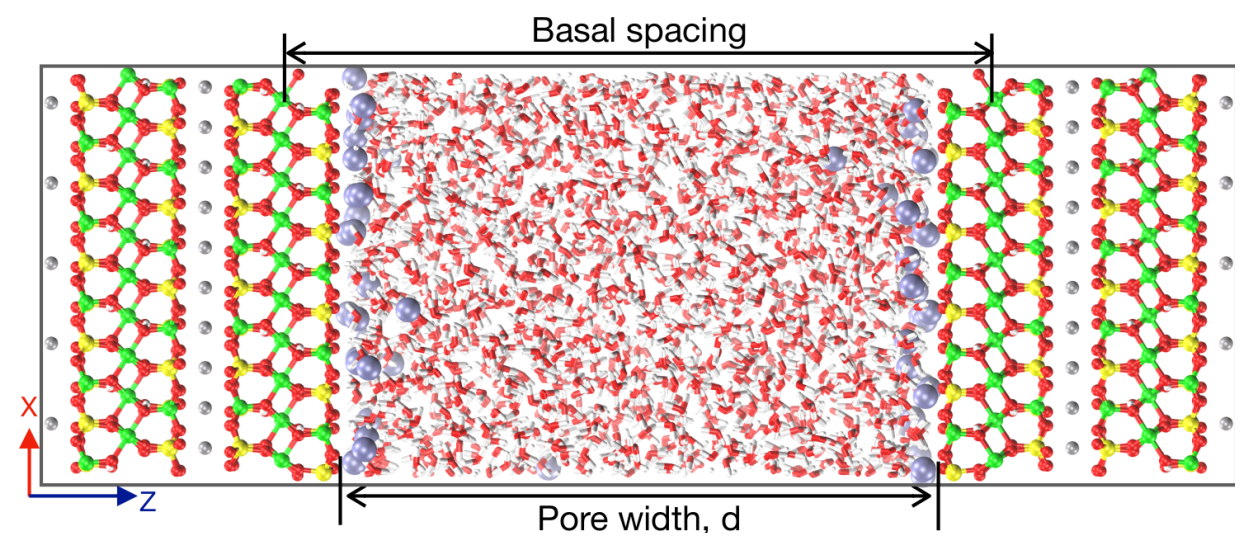

Figure 1: Simulation cell of mica pore containing two mica surfaces (on left and right) confining water molecules, and interstitial cations. The interlayer zone of width $d$ is in equilibrium with bulk-reservoir of water (implicitly) which allows for exchange of water molecules between pore and reservoir. Color legend: Gray - potassium (K), red- Oxygen $(\mathrm{O})$, green - aluminum ( $\mathrm{Al}$ ), yellow - silicon ( $\mathrm{Si}$ ), cyan - exchanged interstitial cation (i.e., either sodium, lithium or hydrogen ion) and water - licorice 


\section{Computational Methodology}

\subsection{Molecular Models of Mica Surface, Water, and Cations}

The muscovite mica supercell containing 4 mica molecules along $z$-direction of thickness 18.13 $\AA$ was repeated to create a mica surface of dimensions $L_{x}=52.048 \AA$ and $L_{y}=54.108 \AA$ $\left(A_{x y}=L_{x} L_{y}=28.16 \mathrm{~nm}^{2}\right)$. The prepared surface contains two exposed surfaces (top and bottom) and one interstitial layer containing $\mathrm{K}^{+}$ions. The M-mica surface was created by

replacing the $\mathrm{K}^{+}$ions of the top exposed surface to $\mathrm{M}^{+}$ion $(\mathrm{M}=\mathrm{Na}$, Li or $\mathrm{H})$. The charge on the framework is located at the exposed silica tetrahedral layer and its magnituded is 1e per unit cell, which is different from MMT studied in literature ${ }^{48,55,58}$ where charge is located either at octahedral, tetrahedral or both layer. The exposed cations are mobile whereas the rest of the mica surface was kept rigid for all simulations and referred here as the mica framework. The mica pore was created by placing the two mica surfaces opposite to each other such that the framework atoms of both surfaces are in registry (Fig. 1). The water molecule was modelled by the three site rigid model and cation by single sphere.

\subsection{Interaction Potential Models}

The van der Waals and electrostatic interactions between two atomistic sites were modelled using Lennard-Jones (LJ) and Coloumbic interactions, ${ }^{68-70}$ respectively . The Coloumbic intearaction were evaluated using three-dimensional (3D) Ewald summation with correction for slab geometry ${ }^{71}$ as described in detail in earlier works. ${ }^{52,67,68,72}$ The short range part of the Ewald summartion and the LJ interactions were evaluated within the spherical cut-off of $14 \AA$. The long-range part of the Ewald summation was evaluated in reciprocal space within the relative accuracy of $10^{-6}$. A vaccum of width $d_{v}=\max \left(d+2 d_{m}, L_{x}, L_{y}\right)$ was added above each mica surface to reduce electrostatic interaction between images, making the entire width of simulation cell in $z$-direction to be $L_{z}=d+2 d_{v}+2 d_{m}$, where $d$ is the pore width and $d_{m}=18.13 \AA$ is the width of mica framework. The mica atoms, water 
molecule, $\mathrm{Na}^{+}$and $\mathrm{Li}^{+}$ion, and $\mathrm{H}^{+}$ion were modelled using CLAYFF, ${ }^{73} \mathrm{SPC} / \mathrm{E},{ }^{74}$ Joung and Cheatham III ${ }^{65}$, and CHARMM $^{75}$ forcefields, respectively. The parameters are given in Table S1 of supporting information (SI). Since, the charge on $\mathrm{H}^{+}$ion is lesser than $\mathrm{Na}^{+}$ and $\mathrm{Li}^{+}$ion, the excess charge on mica framework was managed by increasing the charges of exposed silicon and aluminium atoms of silica tetrahedral layer.

\subsection{Grand Canonical Monte Carlo (GCMC) Simulations:}

The MC simulations were performed in the grand canonical ensemble (at constant $\mu V T ; \mu$ chemical potential of water, $V$ - volume of the interstitial zone and $T$ - temperature). The MC moves performed were, i) displacement, ii) rotation, iii) insertion, and iv) deletion of water molecules and v) displacement of ions with equal probability which were accepted based on Metropolis criteria. ${ }^{69,70}$ The MC simulations were performed at various pore widths in the range of $d=4-40 \AA$. All the simulations were started with an empty pore and a minimum of $10^{9}$ equilibration and $10^{9}$ production moves were performed at $T=298 \mathrm{~K}$. The implicit bulk reservoir corresponds to liquid water at room temperature having density $\left(\rho_{b}=\right) 0.0334$ $\AA^{-3}$ and the chemical potential $(\mu=)-29.37 \mathrm{~kJ} / \mathrm{mol}$, evaluated separately. For the faster equilibration of the simulations, we also performed MD simulations in canonical ensemble (constant $N V T$ ) intermittently during the equilibration period of GCMC simulations. The temperature of the system was maintained using Nose-Hoover thermostat and rigid geometry of the water molecules were maintained using SHAKE algorithm. ${ }^{76}$ All mica farmework atoms were kept frozen by not solving equations of motions for them and simulations were performed for $1 \mathrm{~ns}$ with a timestep of $2 \mathrm{fs}$. The GCMC simulations and property calculations were performed using in-house developed codes and the MD simulations were performed using LAMMPS package. ${ }^{77}$ 


\section{Property Calculations}

The thermodynamics and structure of confined water were analysed by calculating disjoinning pressure $(\Pi)$, swelling free energy $\left(\Delta \Omega^{e x}\right)$, one-dimensional density distribution $\left(\rho_{z}\right)$, orientational distribution $(P(\cos \theta, z))$, pair correlation function $(\mathrm{g}(\mathrm{r}))$ and hydration numebr $\left(C_{n}\right)$ details of some are given below.

\subsection{Disjoining Pressure and Grand Potential}

The fundamental equation which describes the thermodynamics of a confined system is given as,

$$
\mathbf{d} \Omega=-P^{b} \mathbf{d} V-S \mathbf{d} T-N \mathbf{d} \mu+2 \gamma \mathbf{d} A_{x y}-\left(A_{x y} f_{s}\right) \mathbf{d} d
$$

where $P^{b}$ is the bulk water pressure, $V$ is the volume, $S$ is the entropy, $N$ is the number of water molecules adsorbed in the pore, $\gamma$ is the fluid-wall interfacial tension, and $f_{s}$ is the solvation force per unit area of the surface, $A_{x y}$. For the bulk water system, the last two terms are to be removed due to the absence of interface and pore. The excess grand potential $\left(\Omega^{e x}=\Omega-\Omega^{b}\right)$ in differential form is obtained as,

$$
\mathbf{d} \Omega^{e x}=-S^{e x} \mathbf{d} T-N^{e x} \mathbf{d} \mu+2 \gamma \mathbf{d} A_{x y}-\left(A f_{s}\right) \mathbf{d} d
$$

where $S^{e x}=S-S^{b}, N^{e x}=N-N^{b}$, and superscript $b$ indicate a property of a bulk system. At constant $T, \mu$, and $A_{x y}$, we obtain

$$
f_{s}=-\frac{1}{A_{x y}}\left(\frac{\partial \Omega^{e x}}{\partial H}\right)_{T, \mu, A_{x y}}
$$

In literature, ${ }^{52,59,78-82}$ this net force per unit area $\left(f_{s}\right)$ is referred to as the disjoining pressure, $\Pi=P^{z}-P^{b}$, where $P^{z}$ is the normal component of pressure acting on the surface.

Disjoining pressure gives an insight of attraction or repulsion between two surfaces of the slit pore due to pore filling by the surrounding liquid. The negative value of force indicates 
that surfaces are attracted to each other, and the system wants to collapse by squeezing out the confined fluid. Conversely, the positive value of force indicates that the confining fluid pushes the surfaces; hence, squeezing out of it is not favorable but difficult. The confined system would be stable at those pore width where it is in equilibrium with the surrounding fluid (i.e., $\Pi=0$ ). In our simulation, we calculated the disjoining pressure by explicitly calculating the force exerted on the mica framework due to confined water, mobile surface ions, and atoms of opposite side mica framework as,

$$
F=\sum_{i=1}^{N_{\text {surf }}}\left[\sum_{j=1}^{N} f_{i j}+\sum_{j=1}^{N_{\text {ion }}} f_{i j}+\sum_{j=1}^{N_{\text {surf }}} f_{i j}\right]
$$

where $f_{i j}$ is the interatomic force between atom $i$ and $j, N_{\text {surf }}$ and $N_{\text {ion }}$ are the number of (fixed) atoms in the mica framework and free ions in the confined region, respectively. The absolute pressure exerted on the surface is $P^{z}=F(z$-component $) /\left(A_{x y}\right)$, whereas the disjoining pressure is defined as $\Pi(d)=P^{z}(d)-P^{z}\left(d_{\infty}\right)$, where $d_{\infty}$ is the pore system with very large pore-width having bulk-like characteristics.

To obtain the information about the stability of surface separation (at a given $d$ ) and thermodynamics of swelling, we calculate excess grand potential from Eq. 3 using $\Pi$ as $\Delta \Omega^{e x}(d)=\Omega^{e x}(d)-\Omega^{e x}\left(d_{\infty}\right)=A_{x y} \int_{d}^{d_{\infty}} \Pi \mathbf{d} d$. We calculated the total $\Pi$ as well as individual contribution from water $\left(\Pi_{W}\right)$, ions $\left(\Pi_{I}\right)$ and mica framework of other side $\left(\Pi_{S}\right)$ from which total and individual contribution to swelling free energy were also evaluated (i.e., $\Delta \Omega^{e x}, \Delta \Omega_{W}^{e x}, \Delta \Omega_{I}^{e x}$, and $\left.\Delta \Omega_{S}^{e x}\right)$. All the swelling free energy $(\Delta \Omega)$ reported in this manuscript were normalized by thermal energy $\left(k_{B} T\right)$ and area of the mica framework $\left(A_{x y}\right)$, thus the $\Delta \Omega$ has units of $\mathrm{nm}^{-2}$. The numerical value of $\Delta \Omega$ represent the energy required (compared to the thermal energy) to separate clay-minerals of unit area (of $\left.\mathrm{nm}^{2}\right)$. 


\subsection{Denstity Distribution:}

The one dimensional density distirbution of confined water and ions within the pore were evlauated as $\rho_{z}=1 /\left(N_{j} A_{x y}\right) \sum_{i}^{N_{j}} \delta\left(z-z_{i}\right)$ where $N_{j}$ is the total number of atoms (hydrogen, oxygen, or cations), $z_{j}$ is the $z$-coordinates of selected atom, and delta function was evaluated numerically as combination of two discrete heavyside step functions $(\Theta(x)=0$ for $x<0$ and $=1$ for $x>0)$ as $\delta(x)=(1 / d x)(\Theta(x-d x / 2)-\Theta(x+d x / 2))$ where $d x$ is the bin thickness.

\subsection{Orientational Distribution}

We used dipole $\left(e_{D}\right)$ and $\mathrm{HH}$ vector $\left(e_{H H}\right)$ of a water molecule to define the orientation $\left(\theta_{D}\right.$ and $\theta_{H H}$ ) with respect to the (left) mica surface normal $\left(e_{z}\right) \operatorname{as} \cos \theta_{i}=e_{i} \cdot e_{z}$. We calculated the joint probability distribution of water orientation at various location within the pore as $P\left(\cos \theta_{i}, z\right)=\sum_{j}^{N_{j}} \delta\left(\cos \theta_{i}-\cos \theta_{j}\right) \delta\left(z-z_{j}\right)$ which combines the effect of local density of water molecules in the pore. We arbitrarily defined one of the hydrogen atoms as the reference atoms, hence $P\left(\cos \theta_{H H}, z\right)$ is symmetric with respect to the $\cos \theta_{H H}=0$. While plotting, the $P\left(\cos \theta_{i}, z\right)$ were normalised by the maximum value observed for given pore width.

\subsection{Ion Hydration}

To understand the hydration of cations, we evaluated pair correlation function between ion and oxygen of water or mica surface as gI-W/OS $(r)=1 /\left(4 \pi r^{2} N_{I} \rho_{\mathrm{W} / \mathrm{OS}}\right) \sum_{i}^{N_{I}} \sum_{j}^{N_{W} / N_{O S}} \delta(r-$ $r_{i j}$ ), where $r_{i j}$ is the distance between ion and oxygen atom, $\rho_{\mathrm{W} / \mathrm{OS}}$ is the density of water or surface oxygen, $N_{I}, N_{W}$ and $N_{O S}$ are the number of cations, water molecules and surface oxygen atoms, respectively. The total number of water molecules or surface oxygen atoms within the first minima $\left(r_{\text {min }}\right)$ of the $\mathrm{g}_{\mathrm{I}-\mathrm{W} / \mathrm{OS}}(r)$ was evaluated as coordination/hydration number as $C_{n}=\int_{0}^{r_{\min }} \rho_{\mathrm{W} / \mathrm{OS}} 4 \pi r^{2} \mathrm{~g}_{\mathrm{I}-\mathrm{W} / \mathrm{OS}}(r) \mathbf{d} r$. This hydration strucutre of ion was analysed for all cations at each pore width and the $C_{n}$ values for $d_{\infty}$ system was considered as the 
reference value. Instead of reporting the absolute values of $C_{n}(d)$, we report the normalized values as $\mathrm{f}_{i}=C_{n, i}(d) / C_{n, \mathrm{~W}+\mathrm{OS}}\left(d_{\infty}\right) \times 100$, which provides the information on change in hydration structure due to confinement. The subscript $i=\mathrm{W}$, OS and $\mathrm{W}+\mathrm{OS}$ in $\mathrm{f}_{i}$ and $C_{n, i}$ represent the normalised and absolute hydration number of cation due to water, surface oxygen, and total, respectively. During hydration analysis (discussed later) we found sharing of hydration shell where some of the water molecules hydrating a cation also simultaneously hydrates nearby cations. We identified and calculated number of such water molecules which are hydrating only one $\left(C_{n, 1 \mathrm{~W}}\right)$ and simultaneously two $\left(C_{n, 2 \mathrm{~W}}\right)$ cations and reported in normalized form as $\mathrm{f}^{i \mathrm{~W}}=C_{n, i \mathrm{~W}}(d) / C_{n, \mathrm{~W}}\left(d_{\infty}\right) \times 100$. In case of a water molecule hydrating two cations, they could be from the same side of mica framework or opposite sides; percentages of which $\left(\mathrm{f}_{\mathrm{S}}^{2 \mathrm{~W}}\right.$ and $\mathrm{f}_{\mathrm{O}}^{2 \mathrm{~W}}$ ) were also calculated same as $\mathrm{f}^{2 \mathrm{~W}}$. For the $d_{\infty}$ system, the two surfaces are apart and hence $\mathrm{f}_{\mathrm{O}}^{2 \mathrm{~W}}\left(d_{\infty}\right)=0$. We also found (discussed later) that the cation were hydrating only one, simultaneously two or three surface oxygens and for their easy identification, we refer to them as $\mathrm{M}_{i \mathrm{OS}}$ and their percentages are reported as $\chi^{i}(i=1,2$, or 3$)$. To get a three dimensional (3D) picture of the hydration shell of these cations, we calculated the 3D $\mathrm{g}(\mathrm{r})$ within the $r_{\text {min }}$ as, $\mathrm{g}_{\mathrm{I}-\mathrm{W} / \mathrm{OS}}(x, y, z)=1 /\left(N_{I} \rho_{b}\right) \sum_{i}^{N_{I}} \sum_{j}^{N_{W} / N_{O S}} \Theta\left(r_{\text {min }}-r_{i j}\right) \delta\left(x-x_{i j}\right) \delta(y-$ $\left.y_{i j}\right) \delta\left(z-z_{i j}\right)$ normalised by the $\rho_{b}$ for easy comparison across cations.

\section{Results and Discussion}

\subsection{Equilibrium Water Loading}

Figure 2 shows the equilibrium water loading $\left(N_{W}\right)$ in $\mathrm{M}-$ mica $(\mathrm{M}=\mathrm{Na}$, Li, and $\mathrm{H})$ pores obtained from GCMC simulations at various $d$. The water content in all mica systems shows a qualitatively similar trend, however, they are quantitatively different. We observe water molecules of $N_{W} \sim 11,120$ and 120 in Na-, Li-, and H-mica system even at $d=4$ $\AA$, which is the interstitial width of anhydrous (dry) naturally occurring K-mica. This indicates that these ions are hygroscopic in nature which adsorbs water molecules even under 


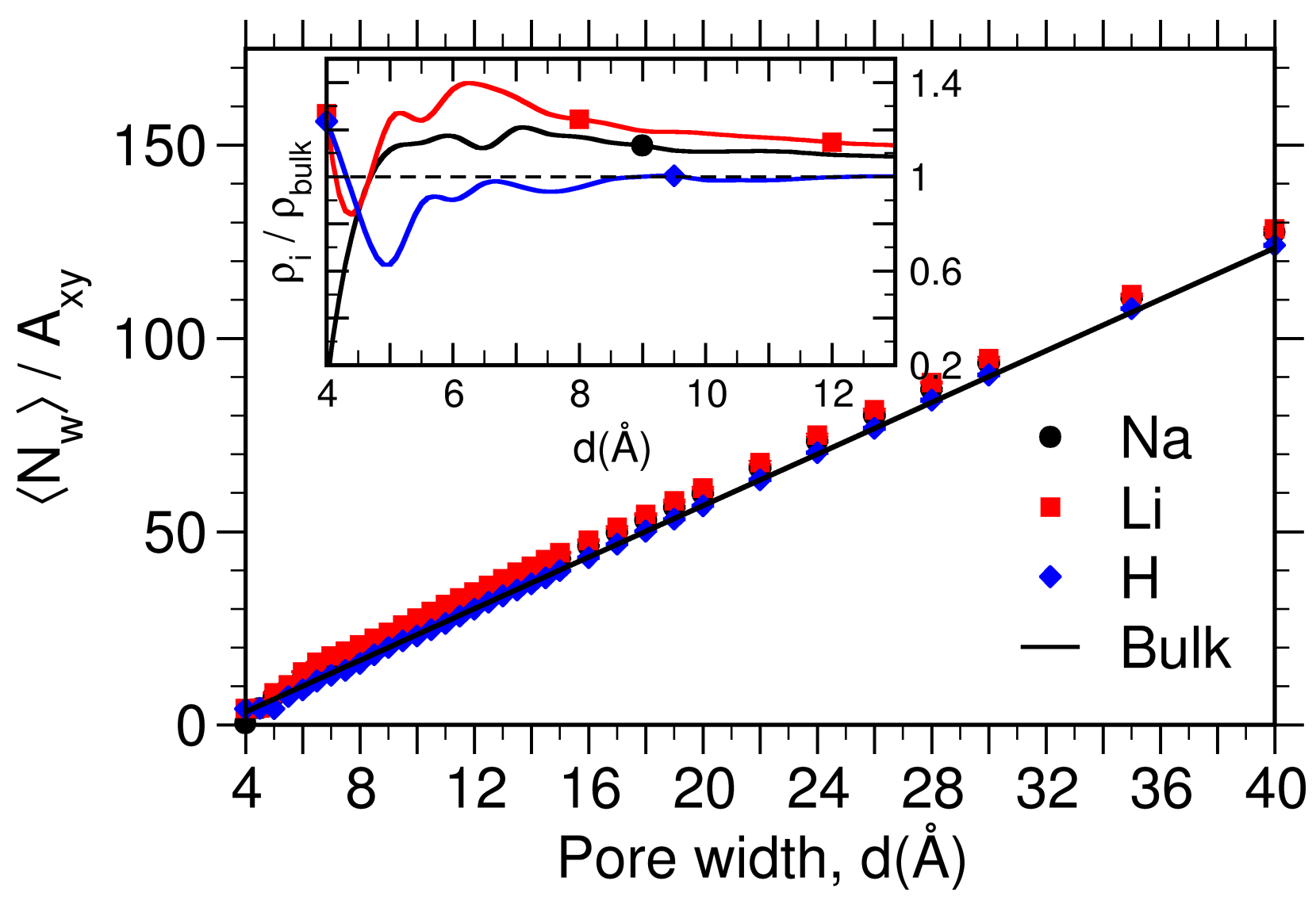

Figure 2: Number of water molecules adsorbed $\left(N_{W}\right)$ in M-mica pore at various pore widths (d) having lateral area of $A_{x y}=28.16 \mathrm{~nm}^{2}$. The solid line is the number based on bulk density of water (i.e., $\rho_{b}=0.0334 \AA^{-3}$ ). Significant deviations are observed as illustrated with clarity in the inset where density of water in confinement $\rho_{i}$ normalized by bulk density $\left(\rho_{b}\right)$ is shown.

extreme confinement. The water loading increases continuously for larger pores of $d>4 \AA$. We observe that the adsorbed water molecules are significantly higher in Na- and Li-mica system than bulk for corresponding $d$ shown by linear line in Fig. 2 (evaluated as $\rho_{b} V_{a v}$, where $V_{a v}=V_{p}-V_{e x c l}$, is the available pore volume, $V_{p}=A_{x y} d$, is the pore volume, $V_{\text {excl }}=A_{x y} \zeta$, is the excluded volume due to rigid wall, and $\zeta$ is the thickness of the excluded zone near mica surface obtained from study of water adjacent to single mica surface (see Fig. S1 of SI) The variation in density of confined water $\left(\rho_{i}=N_{W} / V_{a v}\right)$ with respect to $\rho_{b}$ is better represented in inset of Fig. 2; where we observe significant deviation in $\rho_{i} / \rho_{b}$ form unity at smaller $d$ in the range of $4.5-12 \AA$ and beyond, it approaches unity, asymptotically. This 
clearly signifies that effect of mica confinement is strongly experienced at smaller $d$. Since $\mathrm{H}^{+}$ions have smaller size and higher hydration energy (Table 1) compared to $\mathrm{Li}^{+}$and $\mathrm{Na}^{+}$ ions, we expected that more water molecule would adsorb in the H-mica pores compared to Na- and Li-mica pores. However, we observed a counter-intuitive behavior that $\rho_{i}$ follows the trend of $\rho_{\mathrm{Li}}(d)>\rho_{\mathrm{Na}}(d)>\rho_{b}>\rho_{\mathrm{H}}(d)$.
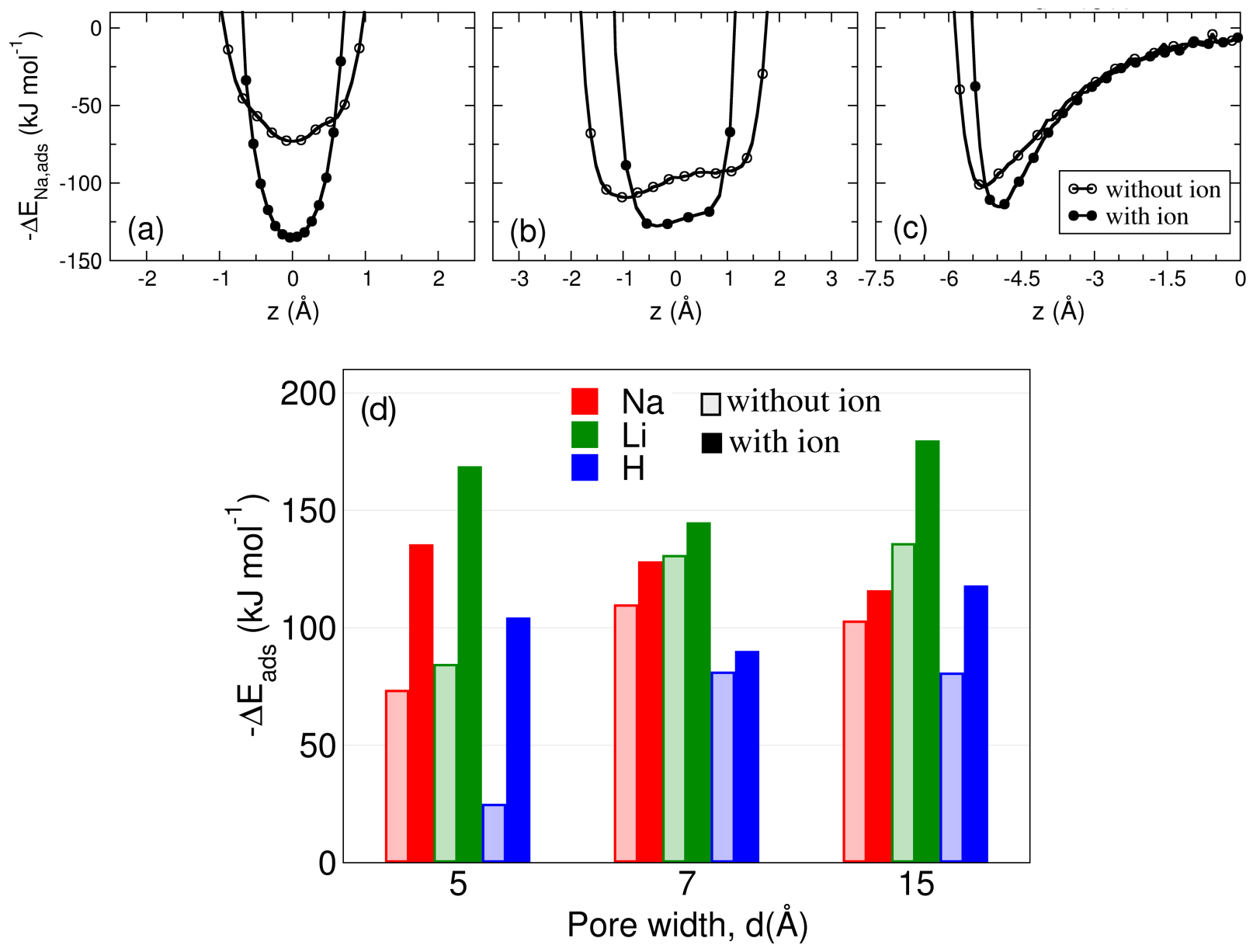

Figure 3: (a-c) Energy of single water molecule calculated at various $z$-position within the Na-mica pore of widths $d=5,7$, and $15 \AA$. The position of probe molecule was selected near the hexagonal cavity such that it is hydrating a cation (with ion) and not-hydrating a cation (without ion), and then interaction energy profile was calculated (see text for details). d) The minima of the water energy profile, referred as adsorption energy $\left(-\Delta \mathrm{E}_{a d s}\right)$, in representative pore widths of M-mica systems.

To understand the reason, we investigated the adsorption energy of water molecules in these confined pores. The prominent adsorption sites of mica clay are the ditrigonal cavity 
(hexagonal type of cavity formed by Si-O-Si bonds) with and without cation, and, regions where water co-hydrate multiple ions simultaneously. Among these adsorption sites, we have calculated the adsorption energy of single water molecules at mica cavity with and without cation for representative pore width. We probed a region of $5 \times 5 \times 2 \AA^{3}$ near a ditrigonal cavity with a grid size of $0.1 \AA$. At each location, the center of mass (oxygen atom) position of water molecule was fixed and $100 \mathrm{MC}$ moves of rotation of water molecule were performed to obtain the minimum energy configuration within this region $\left(x_{m}, y_{m}, z_{m}\right)$. Then we fixed $x, y$ position of water (mainly oxygen) to $x_{m}, y_{m}$ and varied the $z$-position with a step size of $0.1 \AA$. At each $z$-position, $2 \times 10^{4} \mathrm{MC}$ moves of water rotation were performed and the last $10^{4}$ moves were used to obtain average configurational energy of a water molecule. The energy profiles for representative systems studied is shown in Fig. 3a-c. The ion configuration was taken to be the last configuration of GCMC simulations where position of ions are non-uniform due to mobility and adsorption of water molecules. As a result, we do not observe symmetric energy profile at lower $d$ where overlap of interaction due to nearest neighbour is higher. The minimum of this energy profile was considered as the adsorption energy $\left(-\Delta \mathrm{E}_{a d s}\right)$ and the collective data obtained for various ions is shown in Fig. 3d. We observe that the $-\Delta \mathrm{E}_{a d s}$ is higher in mica cavity with ions for all the M-mica studied. Further the trend of $-\Delta \mathrm{E}_{a d s}$ is similar to the trend of $\rho_{i}$, explaining the reason behind observed behavior. Those water molecules which hydrate more than one ion would have significantly higher adsorption energy as compared to single ion case. Hence we did not study those cases and investigated only the limiting cases.

\subsection{Structure of Ions and Water Molecules}

In literature, ${ }^{83-87}$ including our previous work, ${ }^{68,88}$ it has been found that cations tend to adsorb in multiple hydration states at the mineral water interface. Based on surface and water hydration illustrated in Fig. 4, these are classified as i) inner-sphere complexes (IS): where ions directly hydrate the surface oxygen and water molecules, ii) outer-sphere complexes 


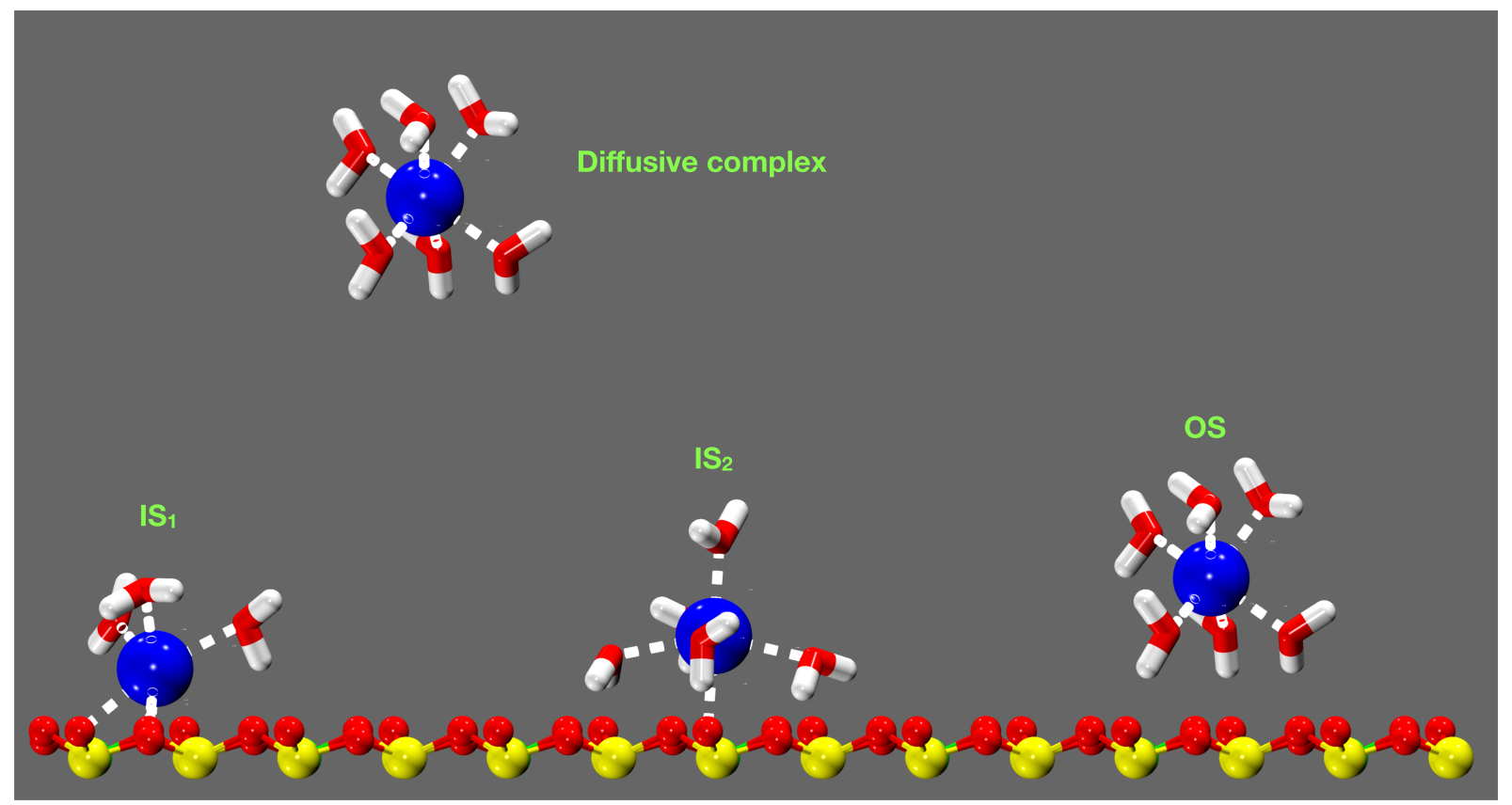

Figure 4: Schematics illustrating the various ion hydration scenarios observed. Inner sphere complex (IS) where cation forms hydration shell with both surface oxygen and water molecules. $\mathrm{IS}_{1}$ is closer to the surface as compare to $\mathrm{IS}_{2}$. Outer sphere complex (OS) where cation is located one water diameter away from surface and interact with the surface via a hydration water molecule, generally, located below. Diffuse ion which does not interact with surface neither directly nor via hydration water.

(OS): where cation is located around one water diameter away from surface and interact via hydration water with the surface, and iii) diffusive complex: where the cation do not interact with the surface, neither directly nor through hydration water. The layering in interfacial water (i.e., location and peak intensity in water density profile) is significantly affected by the type of cations. For the easy identification, WLs are referred to as L1, L2 and so on (smaller numeral indicates WL closer to the surface). Due to confinement, the hydration state of ions and water layering could alter significantly, especially at smaller $d$ due to overlap of interfacial zones and variation in water loading (Fig. 2). We will explain the water and ion structure in mica pore from smaller to larger pore width in Na-mica system first and then discuss Li- and H-mica system. 

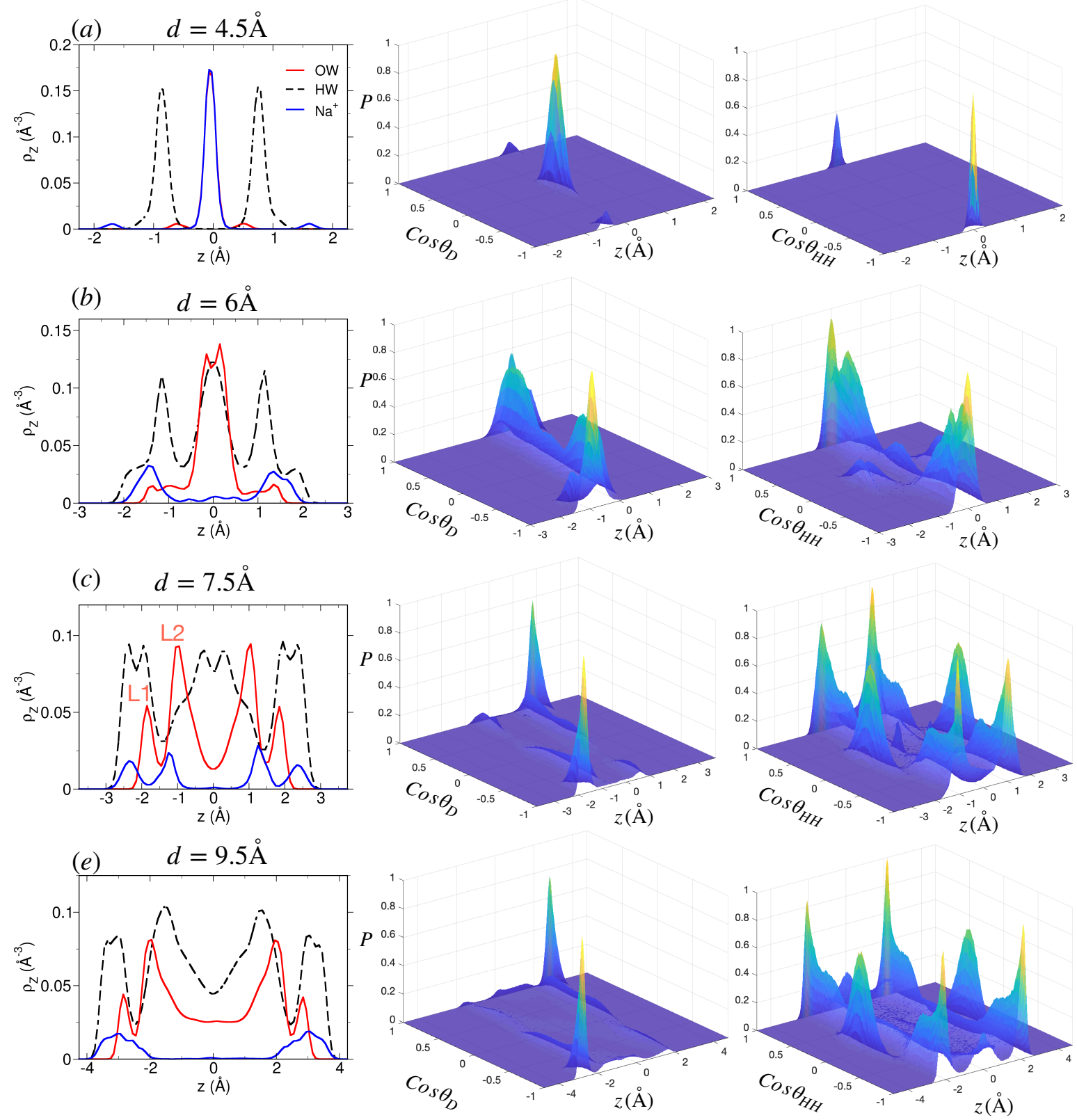

Figure 5: Structure of adsorbed water and cation in the Na-mica pores. (Left column) Density profiles of oxygen (red solid line) and hydrogen (dashed black line) of water molecules, and cations (blue solid line) for various pore widths of Na-mica system. In $d=4.5 \AA$, the oxygen (water) density profile is overlapped by the $\mathrm{Na}^{+}$ion density. Note the redistribution of $\mathrm{Na}^{+}$ions and layering of water with increase in $d . \mathrm{L} 1$ and L2 are the water layers (WLs) located closer to the surface. Probability distribution of $\cos \theta_{D}$ (middle column) and $\cos \theta_{H H}$ (right column) at various $z$-position within the pore. $\theta_{D}$ and $\theta_{H H}$ are angle made by the dipole and $\mathrm{HH}$ vector of water molecules with surface normal. The $P\left(\cos \theta_{i}, z\right)$ reported were normalized by the maximum value observed in that confined system. 


\subsubsection{Structure of Ions and Water in Na-Mica System:}

We found that $\mathrm{Na}^{+}$ion and water density in confined Na-mica pore is significantly different at select pore widths of $d=4,6,7.5$ and $9.5 \AA$ and the pore width in-between these represent the transition zones, which is distinctively captured by our equilibrium thermodynamic simulations. The mica pores of transitions zones are not explained in detail in the chapter; however data is provided as Fig. S2 of SI. At $d=4.5 \AA$, the cations and water molecules, both, are located at the center of the pore forming a single layer (Fig. 5a). The dipole and HH vector orientational distribution of water molecules shows that water molecules are oriented such that hydrogens are pointing towards the surface making a $\cos \theta_{D}=0$ and $\cos \theta_{H H}= \pm 1$. Between $d=4$ to $6 \AA$, the $\mathrm{Na}^{+}$ions migrate towards the surface and at $d=6 \AA$, we observe a cation layer adjacent to each surface with smaller fractions of $\mathrm{Na}^{+}$ions in the center of pore (Fig. 5b). These ions form IS complexes with confined water which forms a central layer; however we observe a split in the water density peak indicating a beginning of formation of separate WLs at higher pore widths. The orientation of water molecules at $d=6 \AA$ exhibits a mix distribution. An intense peak at around $\cos \theta_{D}=0.5-1$ is observed which corresponds to those water molecules that are adsorbed above the hydrating ion having $\cos \theta_{H H}=0.5-1$ due to formation of hydrogen bonds with the surface oxygen aotms. In addition to those, few water molecules hydrate more than one $\mathrm{Na}^{+}$ions simultaneously which gives rise to $\cos \theta_{D}=\cos \theta_{H H} \approx 0$ (Fig. 5b). At $d=7.5 \AA$, the $\mathrm{Na}^{+}$ion density near surface splits into two peaks, albeit, both forming inner sphere complexes with water molecules $\left(\mathrm{IS}_{1}\right.$ is closer to surface than $\mathrm{IS}_{2}$ ). Similarly, the water molecules also exhibits two individual peaks, L1 and L2, adjacent to each mica surface (Fig. 5c). The $P\left(\cos \theta_{D}\right.$ or $\left.\cos \theta_{H H}, z\right)$ exhibits layer-wise orientation of confined water molecules. The L1 water molecules are those which are adsorbed in ion-free cavities of mica surface with hydrogen forming hydrogen bonds with surface oxygen atoms due to which they make $\cos \theta_{D}= \pm 1$ and $\cos \theta_{H H}=0$. The $P\left(\cos \theta_{D}\right)$ of L2 water molecules is much broader and distributed, however, the $P\left(\cos \theta_{H H}\right)$ exhibits strong peaks in the range of $\cos \theta_{H H}=0.25-1$ (Fig. 5c). The reason for the above ob- 
servation is the formation of hydrogen bonds between water molecules of two L2 layer. At $d=9.5 \AA$, we observe the merging of the $\mathrm{IS}_{1}$ and $\mathrm{IS}_{2}$ peaks of $\mathrm{Na}^{+}$ions forming a broader peak having a $2 \AA$ width of the distribution (Fig. 5 d), where ions are found to be in multiple hydration states with surface oxygens (i.e., wider distributions of $\mathrm{Na}^{+}$ions having 1-6 surface oxygen atoms in its hydration shell), as observed in water adjacent to single mica surface shown in Fig. 14 of Adapa et al. ${ }^{68}$ In between $d=7.5-9.5 \AA$, a gradual change in ion density is observed where merging of $\mathrm{IS}_{1}$ and $\mathrm{IS}_{2}$ peaks with formation of ion peak at the center of pore forming OS complexes for $d=8-9 \AA$ pores is also observed (see Fig. S2 of

SI). We observe no significant change in the L1 and L2 layer for $d=7.5-9.5 \AA$; however the width of central bulk-like region develops during these pore widths. Beyond $d=9.5 \AA$, no significant variation in the ion and water density is observed, except that a weaker layer of non-hydrated water molecules (L3) and bulk-like zone (L4) is observed (see Fig. S2 of SI). The L3 layer acts as a buffer layer between structured L2 and bulk-like L4 layer due to which water molecules of the L3 layer have mostly bulk-like behavior albeit a weaker structuring due to formation of hydrogen bonds with water of L2 layer.

\subsubsection{Structure of Ions and Water in Li-Mica System:}

The density distribution of water and cation in Li-mica pore (Fig. 6) is qualitatively similar to that of Na-mica system (Fig. 5). We only report the cases where significant variation from Na-mica system is observed. At $d=4.5 \AA$, we observe that $\mathrm{Li}^{+}$ions are adsorbed mostly at the center of the pore, however, a smaller fraction of ions are adsorbed closer to the surface as well (Fig. 6a). Similarly, three layers of (oxygen) water molecules with majority of them present in the center is also observed. Water molecules hydrating the $\mathrm{Li}^{+}$ions located in the center also have their oxygen atoms located at the center with each hydrogen atoms pointing towards the surface giving rise to $\cos \theta_{D}=0$ and $\cos \theta_{H H}= \pm 1$. The smaller fraction of $\mathrm{Li}^{+}$ ions located near the surface have hydrating water located opposite to the ions with oxygen pointing towards the cation and hydrogen pointing towards the oxygen of mica surface giving 

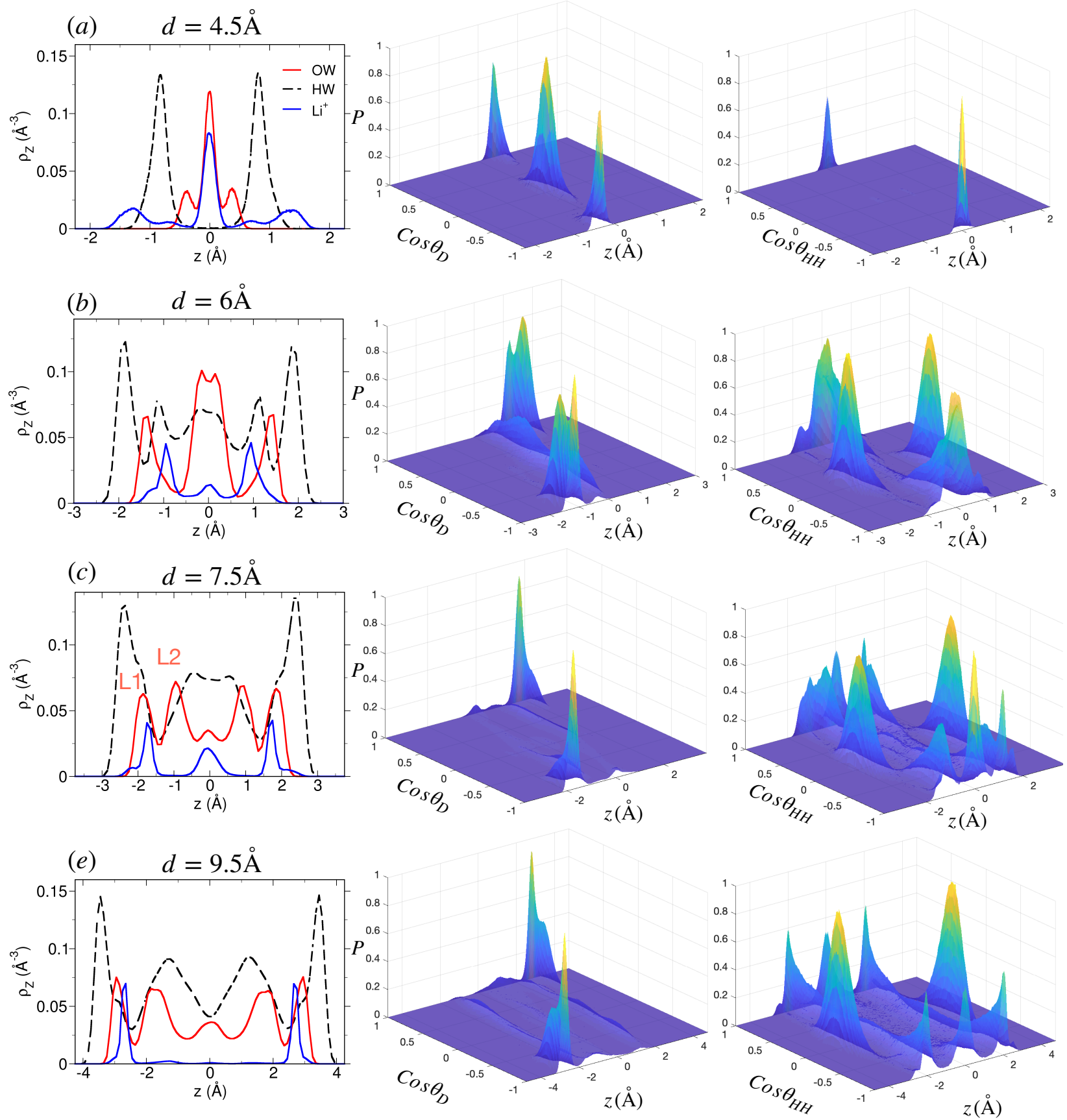

Figure 6: Structure of confined water and cation at various $d$ in Li-mica system. Legends are same as explained in Fig. 5. Note the difference in density of $\mathrm{Li}^{+}$ions and $\mathrm{Na}^{+}$ions of Fig. 5.

rise to $\cos \theta_{D}= \pm 1$. At $d=6 \AA$, we observe three WLs -two L1 layer near each surface and one L2 layer in the middle- and similarly three ion layers - two IS layer near the surface and one OS layer in the middle of the pore (Fig. 6b). A significant fraction of water molecules in 
L1 layer are located closer to the surface compared to $\mathrm{Li}^{+}$ions and also hydrate them which is absent in the Na-mica system. The L1 layer contains both type of water molecules (i.e. hydrating and non-hydrating $\mathrm{Li}^{+}$ions) adsorbed in the cavity with orientation as explained above (i.e., $\cos \theta_{D} \approx \pm 1$ and $\cos \theta_{H H} \approx 0$ ). The orientation of water in L2 layer is similar to that observed in Na-mica at similar pore widths. For $d=6-7.5 \AA$ pores, the water density develops from three (L1-L2-L1) to five (L1-L2-L3-L2-L1) layer configuration (with a small shoulder of L3 layer) due to increased water loading (Fig. 6c) as similarly observed in Na-mica system (Fig. 5b,c); however, no significant change in the $\mathrm{Li}^{+}$ion density is observed as found in $\mathrm{Na}^{+}$ion density at corresponding $d$. We do not observed significant change in orientation of L1 and L2 water molecules from $d=6-7.5 \AA$; however, due to presence of central $\mathrm{Li}^{+}$ion layer, the $P\left(\cos \theta_{H H}\right)$ of L3 layer exhibits a weak structuring. For $d>7.5$ $\AA$, the structure of $\mathrm{Li}^{+}$ion and water is similar to that of Na-mica system, except that the density distribution of $\mathrm{Li}^{+}$ion peak is intense and narrow (Fig. 6d) as compared to $\mathrm{Na}^{+}$ion.

\subsubsection{Structure of Ions and Water in H-Mica System:}

Unlike in Na- and Li-mica systems, the cations in H-mica system are observed as two peaks (adjacent to surfaces) even at narrow pore width of $d=4.5 \AA$ (Fig. 7a). The $\mathrm{H}^{+}$ions are located near one of the surface oxygen atoms of the ditrigonal cavity of one side of the mica framework, whereas, the water molecule is located on the cavity of other side of framework with oxygen pointing towards cation and hydrogens towards surface oxygens $\left(\cos \theta_{D}= \pm 1\right)$. In addition to that we observe a fraction of water molecules having orientation such that $\cos \theta_{D}= \pm 0-0.5$ which is also due to hydration of ions, but due to smaller size of ions having more flexibility in orientation. Between $d=4-6 \AA$, an additional cation density in the center of the pore develops along with increase in loading of water molecules in the center of the pore (Fig. S4 of SI). The orientation of water molecules is governed by the location of $\mathrm{H}^{+}$ion. When the water molecules hydrate the $\mathrm{H}^{+}$ions located closer to the surface, they exhibit distributions in $\cos \theta_{D}$ around \pm 1 and $\cos \theta_{H H} \approx 0$; whereas, those water molecules 

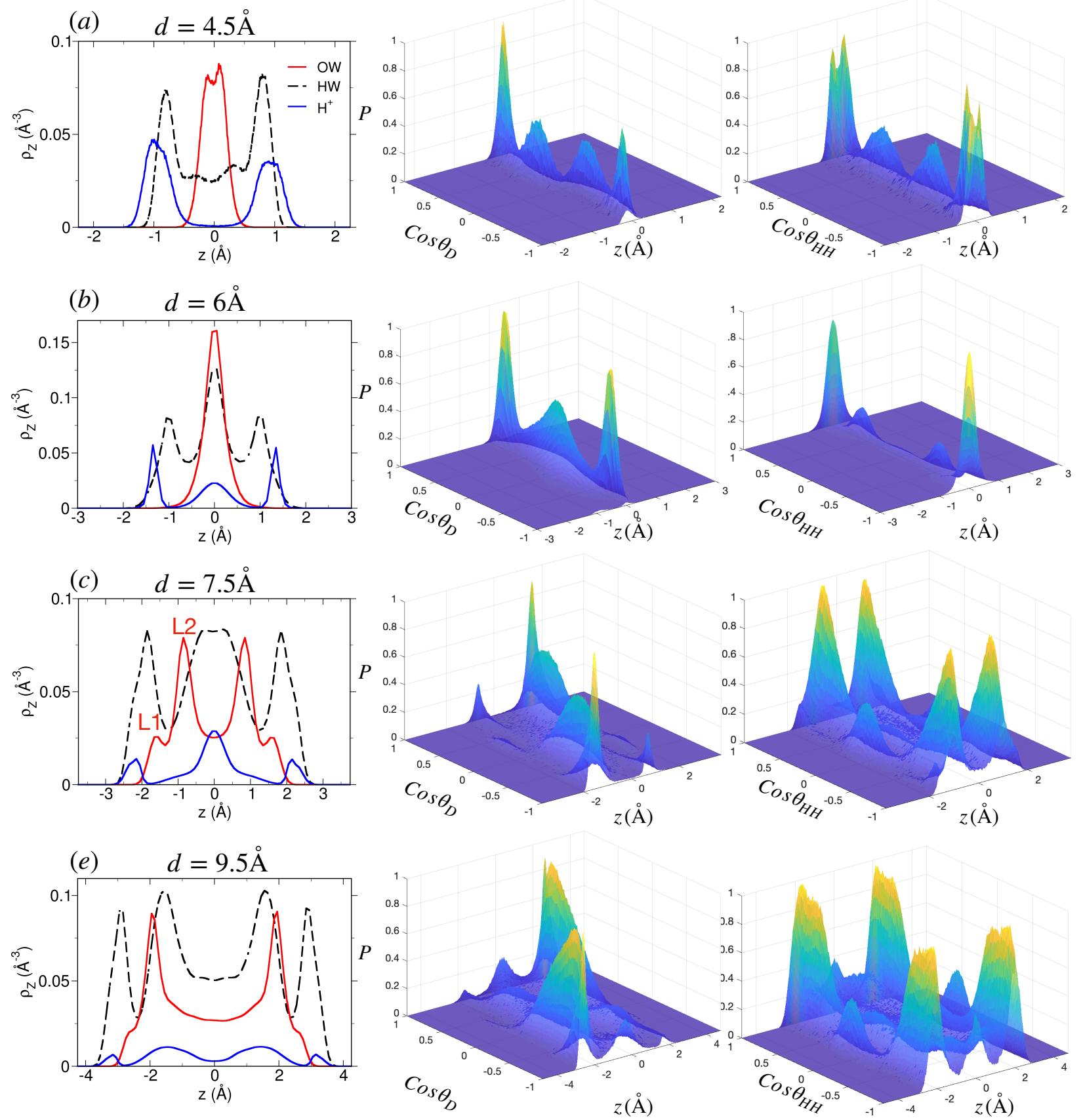

Figure 7: Structure of confined water and cation at various $d$ in H-mica system. Legends are same as explained in Fig. 5. Note the difference in density of $\mathrm{H}^{+}$ions and water compared to Na- and Li-mica system shown in Fig. 5 and 6.

hydrating $\mathrm{H}^{+}$ions located slightly away from the surface exhibit distributions of $\cos \theta_{D} \approx 0.5$ and $\cos \theta_{H H} \approx \pm 1$. The analysis of snapshot indicates that $\mathrm{H}^{+}$ions adsorb near the exposed oxygen atoms of mica framework and not in the center of cavity region as observed for $\mathrm{Na}^{+}$ 
ions reported in earlier work (see Fig. 5 of Adapa et al. ${ }^{68}$ ). At $d=7.5 \AA$, we observe that water molecules are adsorbed in two (L1 and L2) layers adjacent to each surface (Fig. 7c); however the peak intensity of L1 layer is significantly smaller than i) L2 layer of H-mica, ii) L1 layer of Na-mica, and iii) L1 layer of Li-mica system. Further, the $\mathrm{H}^{+}$ion density of the central layer (which forms OS complexes) is higher compared to IS layer near the surface (Fig. 7c). The orientation of $\mathrm{L} 2$ water molecules is mainly due to hydration of $\mathrm{H}^{+}$ions. The density distribution of $\mathrm{H}^{+}$ions is significantly different compared to $\mathrm{Na}^{+}$and $\mathrm{Li}^{+}$ions in the corresponding mica system at similar pore widths. Between $d=7-9.5 \AA$, both ion and water density profile develops which represent the transition states, where the central cation layer breaks down in two individual layer adjacent to each surface forming OS complexes while the width of central bulk-like region of water increases while the densities of L1 and L2 layer remain intact (Fig. S4 of SI). Beyond $d=9.5 \AA$, the development of WLs are similar to Na-mica system, however we observe significant fractions of $\mathrm{H}^{+}$ions in diffuse state (Fig. 4) with uniform density distribution in the central zone of the mica pore. Due to hydration of OS and diffuse $\mathrm{H}^{+}$ions in these mica pores, we observe weak structuring exhibited in $P\left(\cos \theta_{H H}\right)$ in the central region as well.

\subsection{Thermodynamics of Water Adsorption in M-Mica Pores}

Figure 8 shows the obtained disjoining pressure (П) and swelling free energy $\left(\Delta \Omega^{e x}\right)$ profile for Na-, Li- and H-mica system. In previous studies of simple fluids confined between planar surfaces ${ }^{79,89-94}$ the periodic oscillation in $\Pi(d)$ and hence $\Delta \Omega^{e x}$ arises due to sequential change in number of fluid layers. The maxima in $\Pi(d)$ corresponds to compact and dense arrangement of ' $n$ ' fluid layers and subsequent minima in $\Pi(d)$ with both increase or decrease in $d$ leads to formation of diffuse layers of fluids inside pores. Similar behaviour is observed here also, however due to hydrogen bonding capacity of water and hydration of ions these oscillations are not observed at integer multiple of size of the water molecules. The pore widths at which $\Pi=0$ are the configurations where the mica framework are in equilibrium 

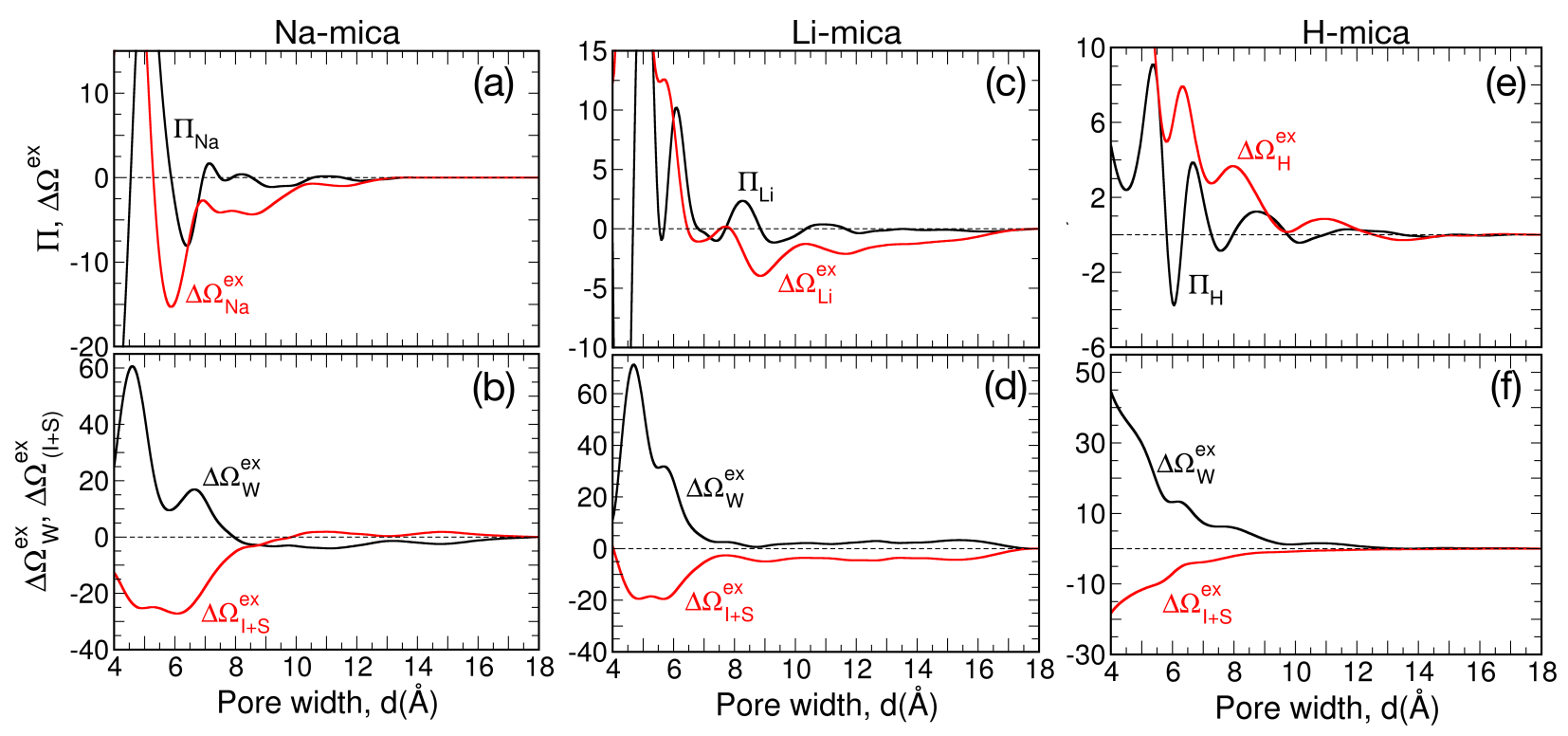

Figure 8: Thermodynamics of water adsorption in (a,b) Na-mica, (c,d) Li-mica and (e,f) H-mica system. (Top row) Disjoining pressure ( $\Pi$ in kbar) and swelling free energy $\left(\Delta \Omega^{e x}\right.$ in $\mathrm{nm}^{-2}$ ) profile observed in M-mica systems. From $\mathrm{Na}^{+}$to $\mathrm{H}^{+}$ion, the $\Delta \Omega^{e x}$ progressively becomes replusive with increase in hydration energy of cations, thus favouring swelling. (Bottom row) Individual contribution to $\Delta \Omega^{e x}$ from confined water $\left(\Delta \Omega_{W}^{e x}\right)$ and cation + mica framework $\left(\Delta \Omega_{I+S}^{e x}\right)$. The long tail in $\Delta \Omega^{e x}$ for $d \geq 11 \AA$ arises due to fluctuations in $\Pi$ which can be assumed to be zero (i.e., $\Pi(d \geq 11 \AA)=0$ ). All values of $\Delta \Omega^{e x}$ (individual contribution and total) were normalized by thermal energy $\left(k_{B} T\right)$ and $A_{x y}$ of mica surface used, thus has units of $\mathrm{nm}^{-2}$. The numerical value represent the energy required, compared to thermal energy, to separate clays of unit area (of $\mathrm{nm}^{2}$ ).

with the confined fluid (reported in Table S2 of SI). For example, these configurations in Na-mica system are found to be $d=5.9(\approx 6), 6.95(\approx 7), 7.5,7.91(\approx 8), 8.5,11.5$, and $\geq 13.5$ $\AA$. At larger pore widths, the two confining surfaces are separated by a pool of bulk-fluid and interfacial effect of surface do not affect, hence $\Pi=0$. Further, the fluctuations in $\Pi$ for $d>11 \AA$ is minimal; hence can be assumed to be zero. Among these configurations, the pore widths which occur at the a) repulsive branch of $\Pi$ (i.e., $\mathbf{d} \Pi / \mathbf{d} d<0$ ) and b) attractive branch of $\Pi$ are the stable and unstable configurations, respectively. These are better illustrated in $\Delta \Omega^{e x}$ profile, where an optima in $\Delta \Omega^{e x}$ (minima for repulsive branch and maxima for attractive branch) is observed for each of these $d$, as $\Pi=-\left(\partial \Delta \Omega^{e x} / \partial d\right)_{\mu, T, A}$. We find that the overall profile of the $\Pi(d)$ and hence $\Delta \Omega^{e x}(d)$, as well as, location of global minimum 
$\left(d_{\min }\right)$ in $\Delta \Omega^{e x}$ is different in Na-, Li- and H-mica system indicating that hydration energy and size of interstitial ions have significant effect on swelling of clay. Further we also observe that the magnitude of $\Delta \Omega^{e x}$ profile shifts upwards (i.e., repulsive interaction between surface increases) with increase in attractive hydration energy of ion in the order of $\mathrm{Na}^{+}, \mathrm{Li}^{+}$, and $\mathrm{H}^{+}$ion. Since the stability of such clay and swelling behaviour is dictated by the global minima in $\Delta \Omega^{e x}$, hence we probe confined system at $d_{\text {min }}$ first, in the order of $\mathrm{Na}^{-}, \mathrm{Li}-$, and H-mica system, then we discuss the $\Delta \Omega^{e x}$ profile.

Table 2: Hydration statistics of cations at $d_{m i n}$ and $d_{\infty}$ for M-mica system.

\begin{tabular}{lllllll}
\hline \hline Property & \multicolumn{2}{c}{ Na-mica } & \multicolumn{2}{c}{ Li-mica } & \multicolumn{2}{c}{ H-mica } \\
\cline { 2 - 7 } & $d_{\min }$ & $d_{\infty}$ & $d_{\min }$ & $d_{\infty}$ & $d_{\min }$ & $d_{\infty}$ \\
\hline$d(\AA)$ & $5.9 \approx 6$ & 26 & $8.85 \approx 9$ & 26 & 15 & 26 \\
$C_{n, \mathrm{~W}+\mathrm{OS}}$ & 6.33 & 6.11 & 4.20 & 4.18 & 2.02 & 2.02 \\
$C_{n, \mathrm{~W}}$ & 3.30 & 3.44 & 3.18 & 3.18 & 1.92 & 1.95 \\
$C_{n, \mathrm{OS}}$ & 3.03 & 2.67 & 1.02 & 1.01 & 0.1 & 0.07 \\
$\mathrm{f}^{1 \mathrm{~W}}$ & 50.45 & 81.48 & 99 & 99.65 & 96.11 & 98.9 \\
$\mathrm{f}_{\mathrm{S}}^{2 \mathrm{~W}}$ & 14.36 & 19.92 & 1 & 0.24 & 2.37 & 1.4 \\
$\mathrm{f}_{\mathrm{O}}^{2 \mathrm{~W}}$ & 32.46 & 0 & 0 & 0 & 0 & 0 \\
$\chi^{0}$ & 0 & 4 & 7 & 5 & 90 & 93 \\
$\chi^{1}$ & 7 & 10 & 85 & 89 & 10 & 7 \\
$\chi^{2}$ & 12 & 24 & 8 & 5 & 0 & 0 \\
$\chi^{\geq 3}$ & 81 & 62 & 0 & 0 & 0 & 0 \\
\hline $\mathrm{f}^{2 \mathrm{~W}}=\mathrm{f}_{\mathrm{S}}^{2 \mathrm{~W}}+\mathrm{f}_{\mathrm{O}}^{2 \mathrm{~W}}$ & & & & &
\end{tabular}

\subsection{Confined Mica Systems at $d_{\min }$}

\subsubsection{Na-Mica System:}

The global minimum is observed at $d_{\min } \approx 6 \AA$ with a very steep minima in the $\Delta \Omega^{e x}$. The analysis of individual contribution of $\Delta \Omega_{W}^{e x}$ and $\Delta \Omega_{I+S}^{e x}$ (Fig. 8b) shows that this minimum is due to strong attraction between interstitial ions and oppositely charged mica framework atoms, while a repulsive free energy interaction is observed due to confined water. We found two major reasons: i) location of ions and water, and, ii) hydration structure of ion from the detailed analysis which are explained one after another. In the structural analysis 
(as discussed in Section 4.2 and Fig. 5), we find that $\mathrm{Na}^{+}$ions are located adjacent to individual surfaces at $d=6 \AA$, while the confined water is present in the center of the pore as single layer. Further, we found that around $90 \%$ of adsorbed water molecules hydrate the $\mathrm{Na}^{+}$ions. To understand comprehensively, we probed in detail the hydration of $\mathrm{Na}^{+}$ ions by surface oxygen atoms and confined water molecules (Table 2). We found that the hydration number of $\mathrm{Na}^{+}$ions is $C_{n, \mathrm{O}+\mathrm{WS}}\left(d_{\min }\right)=6.33$, which is 4 and $6 \%$ higher than $C_{n}$ for $d_{\infty}$ and bulk-water system (Table 1), respectively. Further breakup of $C_{n}$ shows that each $\mathrm{Na}^{+}$ion has an average of $\left(C_{n, \mathrm{~W}}=\right) 3.30$ water molecules and $\left(C_{n, \mathrm{OS}}=\right) 3.03$ surface oxygen atoms. We also calculated the percentage of $\mathrm{Na}$ ions hydrating one, two and three (or more) surface oxygens simultaneously $\left(\mathrm{Na}_{1 \mathrm{OS}}^{+}, \mathrm{Na}_{2 \mathrm{OS}}^{+}\right.$and $\mathrm{Na}_{3 \mathrm{OS}}^{+}$ions) and found them to be 7, 12 and $81 \%$, respectively. We found that $25 \%$ of $\mathrm{Na}^{+}$ions hydrate 4 surface oxygens simultaneously, which are clubbed together and reported as $\mathrm{Na}_{3 \mathrm{OS}}^{+}$ions. The larger size of $\mathrm{Na}^{+}$ions favour formation of hydration shell with more surface oxygen and hence $\mathrm{Na}^{+}$ions fit in the ditrigornal cavities of the mica surface. Indeed the $\mathrm{Na}_{3 \mathrm{OS}}^{+}$ions are located closer to the surface viz-a-viz $\mathrm{Na}_{2 \mathrm{OS}}^{+}$and $\mathrm{Na}_{1 \mathrm{OS}}^{+}$ions. The adsorption of larger percentage of $\mathrm{Na}^{+}$ions near to the mica framework screens electrostatic interactions and thus reduces the the electrostatic repulsion between the framework and therefore increases the overall attractions leading to minimum in $\Delta \Omega_{I+S}^{e x}$ and $\Delta \Omega^{e x}$. Further, the sharing analysis of hydration shell shows that $\mathrm{f}^{2 \mathrm{~W}}=46.82 \%$ out of which $\mathrm{f}_{\mathrm{O}}^{2 \mathrm{~W}}=32.46 \%$ which holds the mica frameworks of opposite sides together by hydration bridge. Similar water bridge by direct hydration of ions located on opposite sides of pore were observed in K-mica system in our recent work, ${ }^{72}$ and others too. ${ }^{56}$ We also calculated $\mathrm{g}_{\mathrm{I}-\mathrm{W} / \mathrm{OS}}(x, y, z)$ of $\mathrm{Na}_{i \mathrm{OS}}^{+}$ions with both water oxygen and surface oxygens as shown in Figure 9. The $\mathrm{g}_{\mathrm{I}-\mathrm{W} / \mathrm{OS}}(x, y, z)$ of $\mathrm{Na}_{3 \mathrm{OS}}^{+}$ions (Fig. 9c) exhibits multiple strong peaks below the ion due to fixed positions of surface oxygens whereas the hydrating water molecules form a broad-distributed corona above the cation. These water molecules are mostly those which are hydrating only one $\mathrm{Na}^{+}$ion at a time. The broad distribution of these ions indicates that the entropy of these molecules is significantly higher than those 
water molecules which are adsorbed in tight zones hydrating multiple ions simultaneously. These $\mathrm{Na}_{3 \mathrm{OS}}^{+}$ions acts as anchor and attracts the other mica surface through water molecules. It was further confirmed by the variation in percentage of $\mathrm{Na}_{3 \mathrm{OS}}^{+}$ion around $d_{\text {min }}$ exhibiting a steep global maxima (Fig. 10), which is responsible for the steep global minima observed in $\Delta \Omega^{e x}$. This indicates that $\Delta \Omega^{e x}$ at $d_{\min }$ is favored by the enthalpic interactions due hydration of $\mathrm{Na}^{+}$ions by surface oxygens predominantly. On the contrary, the $\mathrm{g}_{\mathrm{I}-\mathrm{W} / \mathrm{OS}}(x, y, z)$ of $\mathrm{Na}_{1 \mathrm{OS}}^{+}$ and $\mathrm{Na}_{2 \mathrm{OS}}^{+}$ions exhibits (Fig. 9a,b) multiple peaks below the ion indicating that these $\mathrm{Na}^{+}$ ions are not bound to the surface as strongly as $\mathrm{Na}_{3 \mathrm{O}}^{+}$ions. Further, the peaks of hydrating water molecules are slightly above the $\mathrm{Na}^{+}$ion and majority of these water molecules are hydrating more than one ions simultaneously, especially water molecules hydrating $\mathrm{Na}_{10 \mathrm{O}}^{+}$ ions (Fig. 9a). Due to stronger enthalpic attraction with the two $\mathrm{Na}^{+}$ions, the adsorption position as well as the orientation of these water molecules is restricted, which leads to repulsion in the $\Delta \Omega_{W}^{e x}$, as observed.

\subsubsection{Li-Mica System:}

The global minimum is observed at $d_{\text {min }} \approx 9 \AA$ with $\Delta \Omega^{e x} \sim-4 \mathrm{~kJ} \mathrm{~mol}^{-1} \mathrm{~nm}^{-2}$ due to global minimum in $\Delta \Omega_{W}^{e x}$ while repulsive interaction between ions + framework is observed $\left(\Delta \Omega_{I+S}^{e x}>0\right)$. Due to higher hydration energy and smaller size, the $\mathrm{Li}^{+}$ions form smaller and denser hydration shell with oxygen atoms. Further, the hydrating atoms occupy fixed position relative to each other to prevent repulsive overlap. This is manifested in $\mathrm{g}_{\mathrm{I}-\mathrm{W} / \mathrm{OS}}(x, y, z)$ where narrow and dense peaks at selected locations are observed, unlike distributed regions observed in hydration shell of $\mathrm{Na}^{+}$ions (Fig. 9). The smaller size of $\mathrm{Li}^{+}$ions affect i) the water hydration, ii) water sharing between two hydration shell and iii) surface hydration which is manifested in hydration statistics (Table 2). We find that $C_{n, \mathrm{O}+\mathrm{WS}}\left(d_{\min }\right)=4.20$, significantly lesser than $C_{n}$ of $\mathrm{Na}^{+}$ion, out of which $C_{n, \mathrm{~W}}=3.18$. Further, the percentage

of water molecules sharing the hydration shell is (i.e., $\left.\mathrm{f}^{2 \mathrm{~W}}=\right) 1 \%$ only; and that too with the cations of the same side (i.e., $\mathrm{f}_{\mathrm{S}}^{2 \mathrm{~W}}=\mathrm{f}^{2 \mathrm{~W}}$ ). The remaining hydrating water molecules are not 

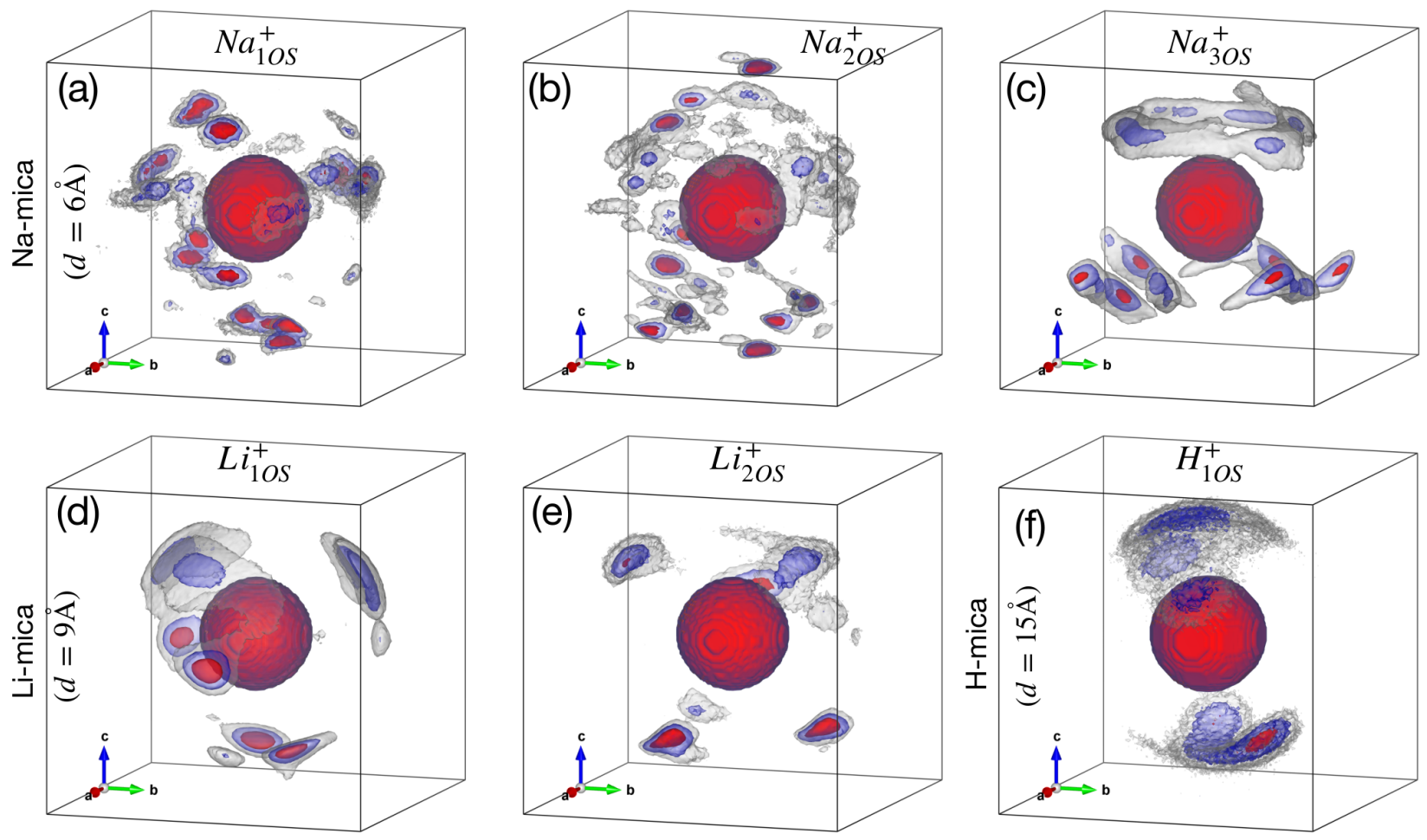

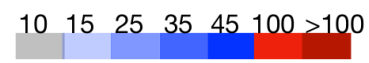

Figure 9: Hydration structure of cations in M-mica systems. Three-dimensional ion-oxygen (water + mica) pair correlation function, $\mathrm{g}_{\mathrm{I}-\mathrm{W} / \mathrm{OS}}(x, y, z)$, evaluated within the $r_{\min }$ for cations hydrating only one $\left(\mathrm{M}_{1 \mathrm{OS}}^{+}\right)$, simultaneously two $\left(\mathrm{M}_{2 \mathrm{OS}}^{+}\right)$and three (or more, $\mathrm{M}_{3 \mathrm{OS}}^{+}$) surface oxygens. The local density in $\mathrm{g}_{\mathrm{I}-\mathrm{W} / \mathrm{OS}}(x, y, z)$ was normalised by $\rho_{b}$ for easy comparison across all cases. Iso-surfaces for selected values are shown by different semi-transparent colours (legend at bottom left). For cations, the $\mathrm{g}_{\mathrm{I}-\mathrm{W} / \mathrm{OS}}(x, y, z)$ peaks above the cation is due to water and below due to oxygens of mica. When surface hydration is more and stronger (in $\mathrm{Na}_{3 \mathrm{OS}}^{+}$), the water hydration is mobile and distributed forming corona above cation. Conversely, when hydration water is strongly adsorbed near $\mathrm{Na}_{1 \mathrm{OS}}^{+}$and $\mathrm{Na}_{2 \mathrm{OS}}^{+}$ions, the surface adsorption is lesser and diffuse. The $\mathrm{g}_{\mathrm{I}-\mathrm{W} / \mathrm{OS}}(x, y, z)$ peaks just below the equator of $\mathrm{Li}_{1 \mathrm{OS}}^{+}$ ion is due to water hydration. The distributed peak below $\mathrm{H}_{1 \mathrm{OS}}^{+}$ion is due to mobility of cation.

shared and hydrate single ion alone (i.e., $\mathrm{f}^{1 \mathrm{~W}}=99 \%$ ). Since, surface oxygen atoms are fixed in framework based on chemistry of surface, which does not coincide with the hydration location of $\mathrm{Li}^{+}$ions, hence the probability of multiple surface oxygen atoms hydrating $\mathrm{Li}^{+}$ ions decreases. This is manifested in the fraction of $\mathrm{Li}_{2 \mathrm{OS}}^{+}$and $\mathrm{Li}_{3 \mathrm{OS}}^{+}$ions which are found to be only 8 and $0 \%$, unlike higher fractions observed in Na-mica system (Table 2). Hence, the total hydration with surface decreases to $C_{n, \mathrm{OS}}=1.02$, where $85 \%$ of $\mathrm{Li}^{+}$ions hydrate 
single oxygen atom. When we compare these hydration statistics with $\mathrm{Li}^{+}$hydration for $d_{\infty}$ system (Table 2), the numbers are very close, suggesting that no significant change occurs in their hydration structure for $d_{m i n}$ as well. These numbers are significantly smaller than observed for Na-mica systems at global minima (Table 2). In the calculated $\mathrm{g}_{\text {I-W/Os }}(x, y, z)$, the peaks located below the ion corresponds to surface oxygen whereas peaks just below the equator corresponds to L1 water hydrating the ions. The L2 water mostly hydrate above the ions and they also form intense and narrow 3D peaks confirming that hydrating structure is very tight. Due to limited sharing, each ion behaves as individual moiety on the surface covered by the water molecule and anchored by the surface oxygens. Further, at $d_{\min }$, we observe a smaller but significant L3 layer (Fig. 6) which contains mostly non-hydrated water molecules which acts as bridging layer forming hydrogen bonded network between hydrated and non-hydrated water molecule of the system present on individual surfaces. Due to this energetically favored configuration, we observed a minima in $\Delta \Omega_{W}^{e x}$. The repulsion in $\Delta \Omega_{I+S}^{e x}$ is due to dipole-dipole interaction between mica framework atoms along with surface ions.

\subsubsection{H-Mica System:}

The $\Delta \Omega^{e x}$ profile is repulsive at smaller pore widths and approaches to zero at larger pore widths due to small fluctuations in $\Pi$ (Fig. 8e). This suggest that formation of thick water layer between H-mica surface is favourable and stable. We considered $d=15 \AA$ as the significantly larger pore width where the hydration structure of $\mathrm{H}^{+}$ion is analyzed. The size of $\mathrm{H}^{+}$ion is even smaller than $\mathrm{Li}^{+}$ion due to which its hydration number is only $\left(C_{n}=\right) 2$, and we found that it remains constant at all $d$. Further, we found that only $10 \%$ of $\mathrm{H}^{+}$ ions remain close to surface having one surface oxygen in the hydration shell whereas rest of the atoms are present as diffuse ion spanning the entire width of the pore. We analyzed $\mathrm{g}_{\mathrm{I}-\mathrm{W} / \mathrm{OS}}(x, y, z)$ of $\mathrm{H}_{1 \mathrm{OS}}^{+}$ions and found one strong yet distributed peak each above and below the ions due to water and surface oxygen, respectively. Due to smaller size and lesser hydration number, these ions do not acts as anchor as like $\mathrm{Na}^{+}$and $\mathrm{Li}^{+}$ions to hold the 
mica surfaces together. Being attached to surface decreases their entropy while maintaining the same hydration number does not favor enthalpically either. Conversely, being in water zone with same hydration number increases its entropy, hence leads to decrease in $\Delta \Omega_{W}^{e x}$ with increase in $d$.

The above analysis indicates that those ions which has higher size that snuggly fits and forms larger number of hydration with surface oxygens of mineral surface acts as anchor and holds the opposite side mineral surface by forming water bridge. Such scenario is observed recent study of water adsorption in in $\mathrm{K}-, \mathrm{Rb}$ - and Cs-mica pres. ${ }^{72}$ With decrease in size of ion and increase in hydration energy, the interaction with mineral surface decreases and ion hydration is favored by free water molecules, therefore such ions favor swelling in mineral surfaces.

\subsection{Analysis of $\Delta \Omega^{e x}$ Profile of M-Mica Systems}

\subsection{1 $\Delta \Omega^{e x}$ of Na-Mica System:}

We now focus on the overall $\Delta \Omega^{e x}$ profile to understand the reason for the observed behaviour (Fig. 8a,b). To probe the system in detail, we divide the $\Delta \Omega^{e x}$ profile of Na-mica system into subzones of $d<6,6-8.5,8.5-10.5$ and $>10.5 \AA$. The repulsion in $\Delta \Omega^{e x}$ for $d<6 \AA$, is observed due to decrease in hydration of $\mathrm{Na}^{+}$ions as shown in Fig. 10a. Both, the number of water molecules hydrating the ions $\left(f_{\mathrm{W}}\right.$ in Fig. 10a) as well as fractions of $\mathrm{Na}_{3 \mathrm{OS}}^{+}$ions $\left(\chi^{\geq 3}\right.$ in Fig. 10c) decreases leading to decrease in enthalpic attraction resulting in repulsion in $\Delta \Omega_{W}^{e x}$ and $\Delta \Omega_{I+S}^{e x}$ (Fig. 8). The regime between $d=6-8.5$ corresponds to confined system between two successive minima in $\Delta \Omega^{e x}$ with a deep minima at $d=6 \AA$, significant maxima at $d=7$ $\AA$, and broader minima at $d=8.5 \AA$. Figure $10 \mathrm{a}-\mathrm{c}$ of the ion hydration shows that during this regime a significant change in hydration behavior from surface and water molecules occurs which is responsible for the observed behavior. The detailed analysis at $d_{\text {min }}=6 \AA$, reported in previous section, has shown that the minima in $\Delta \Omega^{e x}$ occurs primarily due to maximum hydration of $\mathrm{Na}^{+}$ions with surface oxygen. The maxima in $\Delta \Omega^{e x}$ at $d=7 \AA$ occurs due 

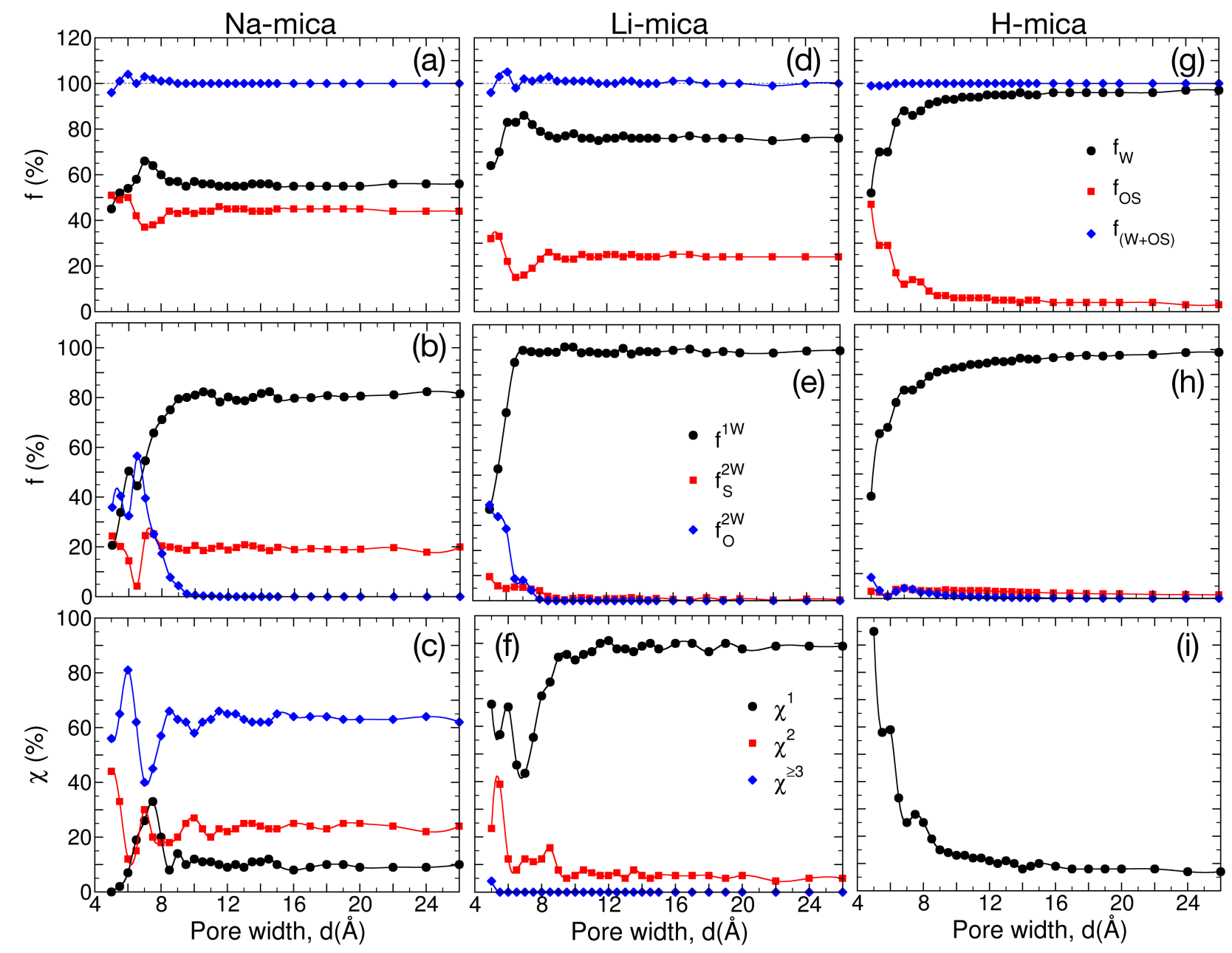

Figure 10: Detailed analysis of (top row) hydration shell, (middle row) water sharing, and (bottom row) surface hydration of (a-c) $\mathrm{Na}^{+}$, (d-f) $\mathrm{Li}^{+}$, and $(\mathrm{g}-\mathrm{i}) \mathrm{H}^{+}$ions in respective $\mathrm{M}-$ mica system. (Top row) Variation of normalized hydration number due to water $\left(f_{W}-\bullet\right)$, oxygen of surface $\left(\mathrm{f}_{\mathrm{OS}}-\mathbf{}\right)$, and total $\left(\mathrm{f}_{\mathrm{W}+\mathrm{OS}}-\boldsymbol{)}\right)$ with respect to $d$. (Middle row) Percentage of water molecules hydrating only one cation (i.e., no sharing, $\mathrm{f}^{1 \mathrm{~W}}-\bullet$ ), two cations of same side (i.e., lateral sharing, $\mathrm{f}_{\mathrm{S}}^{2 \mathrm{~W}}-\mathbf{\square}$ ), and two cations of opposite side (bridge formation, $\left.\mathrm{f}_{\mathrm{O}}^{2 \mathrm{~W}}-\bullet\right)$. (Bottom row) Percentage of cations hydrating only one surface oxygen $\left(\chi^{1}-\bullet\right)$, simultaneously two surface oxygens $\left(\chi^{2}-\mathbf{\square}\right)$, and three (or more) surface oxygens $\left(\chi^{\geq^{3}-}\right)$. Percentage of cations forming OS complexes or diffuse ions are $\chi^{0}=100-\sum_{i>0} \chi^{i}$.

to maximum hydration by water molecules $\left(\mathrm{f}_{\mathrm{W}}=66 \%\right)$ and minimum hydration by surface oxygens $\left(f_{\mathrm{OS}}=37 \%\right)$ especially by the significant decrease in percentage of $\mathrm{Na}_{3 \mathrm{OS}}^{+}$ions (Fig. 10a,c). The structural analysis shows that between $d=6-7.5 \AA$, water and ions change from single layer to compact double layer with rearrangement of water completing at $d=7$ $\AA$ (Fig. 5) followed by $\mathrm{Na}^{+}$ions at $d=7.5 \AA$. This transition is also reflected in $\Delta \Omega_{W}^{e x}$ which 
shows a local maxima in $d \approx 7 \AA$ due to structuring of water, whereas continuous increase in $\Delta \Omega_{I+S}^{e x}$ is observed due to increased distance between mica surfaces and re-structuring of ions. Further, the variation in $\Delta \Omega_{W}^{e x}$ and $\Delta \Omega_{I+S}^{e x}$ is continuous between $d=7-8.5 \AA$ with opposite effect (i.e., increase in attraction and repulsion, respectively) resulting in minima at $\Delta \Omega^{e x}$ at $d=8.5 \AA$. The $\mathrm{Na}^{+}$ion hydration by water and surface oxygen is recovered during $d=7-8.5 \AA$ by the adsorption of water molecules and restructuring which gives rise to $f_{\mathrm{OS}}=44 \%$ and $\chi^{\geq 3}=66 \%$ (Fig. 10a,c). These analysis reconfirm above observation that $\mathrm{Na}^{+}$ions acts as anchor by forming multiple hydration with surface oxygen molecules to hold the mica surface together with forming bridge by the confined water molecules. Further, the hydration of ions shows no significant change in individual and total behavior beyond $d=8.5 \AA$. The regime between $d=8.5-10.5 \AA$, shows an increase in $\Delta \Omega^{e x}$ with a local maxima at $d=10.5 \AA$ while $\Delta \Omega_{W}^{e x}$ exhibits a broad, continuous and attractive minima. The structural analysis of confined system shows development of L3 layer from a non-singificant to fully developed layer, which acts as interfacial layer forming hydrogen bonded network across the pore widths by the adsorption of non-hydrated water molecules mainly. Beyond $d=10.5 \AA$, the system $\Delta \Omega^{e x}$ reaches to zero asymptotically due to adsorption of bulk-like water molecules in the central zone, as evident from the change in structure of water and $\Delta \Omega_{W}^{e x}$.

\subsection{2 $\Delta \Omega^{e x}$ of Li-Mica System:}

Similar to the Na-mica system, we also observe multiple minima in the $\Delta \Omega^{e x}$ for the Limica system (Fig. 8c). The major difference is that we observe four distinct minima (at $d_{1}=5.5, d_{2}=6.85 \approx 7, d_{3}=8.85 \approx 9$ and $d_{4}=11.65 \AA$ ) with global minimum at third minima (i.e., $d_{\min }=d_{3}$ ). To elucidate the reason behind these minima in $\Delta \Omega^{e x}$, here also we divided overall profile into sub-regimes: i) $d \leq 5.5$, ii) $d=5.5-7$, iii) $d=7-9$, and iv) $d>9 \AA$. The local minima at $d_{1}=5.5 \AA$ in $\Delta \Omega^{e x}$ is repulsive and occurs due to attractive contribution from $\Delta \Omega_{I+S}^{e x}$ and repulsive contribution from $\Delta \Omega_{W}^{e x}$ (Fig. 8d). The $\mathrm{Li}^{+}$ions have 
$C_{n}\left(d_{1}\right)=4.29$ out of which $33 \%$ contribution arises from surface oxygen and the rest from water molecules. Further, only $5 \%$ of $\mathrm{Li}^{+}$ions do not form hydrate surface oxygen whereas 57 and $39 \%$ of ions have respectively one and two surface oxygens in their hydration shell. Among the various pore widths, the $\mathrm{Li}^{+}$ions have highest surface hydration at $d_{1}=5.5 \AA$, which gives rise to highest attraction in $\Delta \Omega_{I+S}^{e x}$. When we compare the behaviour of $\Delta \Omega_{I+S}^{e x}$ between Na- and Li-mica system, the qualitative behaviour is similar, however, it is more attractive in Na-mica than Li-mica system due to hydration with surface oxygens. At similar pore width, the $\mathrm{Na}^{+}$ions have $C_{n, \mathrm{OS}}=3.03$ surface oxygen with $\chi^{\geq 2}=98 \%$; whereas these numbers are significantly lesser -around 1.37 and 39\%, respectively- in Li-mica system. The observed repulsion in $\Delta \Omega_{W}^{e x}$ (at $d_{1}$ ) is due the arrangement of water molecules in confined pore, such that oxygens form single central layer with positively charged hydrogens pointing towards the positively charged mica framework (Fig. 5). For $d<5.5 \AA$, the water adsorption and hence the hydration decreases which gives rise to repulsion in $\Delta \Omega_{W}^{e x}$ and hence $\Delta \Omega^{e x}$, as observed (Fig. 8c,d).

The minimum in $\Delta \Omega^{e x}$ at $d_{2}$ is due to attractive minimum in $\Delta \Omega_{W}^{e x}$ and repulsive maxmimum in $\Delta \Omega_{I+S}^{e x}\left(d \approx d_{2}\right)$, which is completely reversal to the behvaior observed at $d_{1}$ (Fig. 8c,d). The main reason is the redistribution of $\mathrm{Li}^{+}$ions forming an additional central layer where $\mathrm{Li}^{+}$ions form an OS complexes with the water molecules (Fig. 6). Due to this redistribution, $\left(\chi^{0}=\right) 45 \%$ of ions do not hydrate surface oxygen and hence leads to significant dip in surface hydration (from $\mathrm{f}_{\mathrm{OS}}=33$ to $16 \%$ ) and increase in water hydration (from $\mathrm{f}_{\mathrm{W}}=70$ to $86 \%$ ) while total hydration number is similar (Fig. 10d-f). This change in hydration is manifested in the $\Delta \Omega_{W}^{e x}$ and $\Delta \Omega_{I+S}^{e x}$. Between $d=5.5-7 \AA$, adsorption of water molecules leads to transition from single WL in the center to L1 and L2 layers at each surface with a transition at $d=6 \AA$, where local maxima in the $\Pi(d)$ is observed due tight packing of WLs in L1-L2-L1 arrangment. In the third regime of $d=7-8.5 \AA$, we once again observe a redistribution of ions where reverse trend of $\mathrm{Li}^{+}$ion migration from the center of the pore to near the mica surface is observed along with development of L1, L2 layer and 
common L3 WL at the center of pore. Due to migration of ions, the $\chi^{0}$ decreases from 45 to $7 \%$, which gives rise to local minima in $\Delta \Omega_{I+S}^{e x}$ at $d_{3}$. Further, we observed that all the additional water molecules adsorbed during this regimes are non-hydrated water molecules, hence the hydration of ions is not affected significantly. Only those water molecules which were hydrating more than one ions simultaneously, their number decreases due to formation of individual hydration shell around each ions. Therefore, no significant change in $\Delta \Omega_{W}^{e x}$ is observed during this regime. For $d>9 \AA$, there are still small fraction of $\mathrm{Li}^{+}$ions which are present away from the surface and with increase in pore width and adsorption of water molecules they migrate towards the surface. This leads to $\Delta \Omega_{I+S}^{e x}$ and $\Delta \Omega_{W}^{e x}$ approaching to zero asymptotically with local fluctuations in ion and water $\Delta \Omega^{e x}$, which leads to local minima in $\Delta \Omega^{e x}$, as observed.

\subsection{3 $\Delta \Omega^{e x}$ of H-Mica System:}

The $\Delta \Omega^{e x}$ profile is repulsive at smaller pore widths and approaches to zero at larger pore widths due to small fluctuations in $\Pi(d)$. The individual contribution of $\Delta \Omega_{I+S}^{e x}$ and $\Delta \Omega_{W}^{e x}$ are, respectively, attractive and repulsive in nature and asymptotically approaches to zero at larger $d$. The reason for these observations could be understood from the hydration behavior of $\mathrm{H}^{+}$ions. At all $d$, we observe that $\mathrm{C}_{n}$ of $\mathrm{H}^{+}$ions is constant and no significant increase due to surface hydration is found as observed in $\mathrm{Na}^{+}$and $\mathrm{Li}^{+}$ions. Further, due to smaller size of $\mathrm{H}^{+}$ions they hydrate only two oxygens (of water or one water and one surface oxygen). By adsorbing to the surface oxygen, the $\mathrm{H}^{+}$ions do not gain an extra enthalpic contribution, while looses mobility and hence entropy. Therefore, from the free energy perspective, hydration of $\mathrm{H}^{+}$ions in bulk region of confined system is favorable. As a result, we observe that most of the ions migrate to center of the bulk and found as diffuse ions at large $d$. Therefore hydration of $\mathrm{H}^{+}$ions with surface decreases (and conversely with water increases) with increase in $d$. The reason for $\Delta \Omega_{W}^{e x}$ being repulsive is that when we decrease the pore width, we are increasing the concentration of distributed $\mathrm{H}^{+}$ions in the confined 
system which increases the osmotic pressure. Also, the decrease in $d$ brings the $\mathrm{H}^{+}$ions closer to the surface, thus screening the electrostatic interactions and hence leading to continuous attraction between mica frameworks resulting in $\Delta \Omega_{I+S}^{e x}<0$. The local minima in $\Delta \Omega^{e x}$ arises due to minor fluccuations in $\Delta \Omega_{W}^{e x}$ and $\Delta \Omega_{I+S}^{e x}$ which corresponds to development of $\mathrm{H}^{+}$ions as IS, OS and diffuse ions. As mentioned earlier that oscillations in $\Pi$ (and therefore $\left.\Delta \Omega^{e x}\right)$ occurs due to transition from 'n' ordered to - 'n' diffuse to - 'n+1' ordered layers in the confined fluid. Here also we observe similar behavior of formation of layers in ions and water. Below $d=6 \AA$, the depletion of water molecules from hydration shell of $\mathrm{H}^{+}$ions leads to repulsion in $\Delta \Omega_{W}^{e x}$ and hence $\Delta \Omega^{e x}$. At $d=6 \AA$, the $\mathrm{H}^{+}$ions are arranged to from IS and OS complexes with water molecules adsorbed in the center of the pore having majority of $\mathrm{H}^{+}$ion fomring IS complexes. The complete hydration and diffuse water layer gives rise to local minimum in $\Delta \Omega^{e x}$. Between $d=6-7.5 \AA$, we see the transition in ions and water arrangement and at $d=7.5 \AA$, the $\mathrm{H}^{+}$ions are arranged such that majority of them (around 70\%) are forming OS complexes with two water layers formed adjacent to each mica surface. Similarly, for $d=7.5-10 \AA$, we observe the transition of central one OS cation layer to formation and development of two OS cation layer (one layer adjacent to each surface) along with development of interfacial water layer. At the completion of this OS cation layer formation, we observe the minima in $\Delta \Omega^{e x}$ at $d \approx 10 \AA$. For $d>10 \AA$, the $\mathrm{H}^{+}$ ions mostly migrate to the center of pore due to favorable hydration with water molecules which gives rise to slow approach of $\Delta \Omega^{e x}$ to zero.

\section{Summary and Conclusion:}

Here we focus on understanding the role of different hydration properties of cation on thermodynamics of clay swelling by water adsorption. The clays acquire cations of different properties by cation exchange, which plays a central role in many applications such as nutrient supply in soil, oil extraction, disposal of nuclear waste, cosmetics and pharmaceuticals. 
The swelling capacity and stability of clay after exchange of cation of different hydration properties will change significantly and hence fundamental understanding of clay swelling is necessary for their efficient utilisation. For this we have chosen mica as the reference clay and $\mathrm{Na}^{+}, \mathrm{Li}^{+}$, and $\mathrm{H}^{+}$ions as the interstitial cations, which has significant differences in hydration properties. We performed several GCMC simulations at various pore widths $(d=4-40 \AA)$ to probe water adsorption. The swelling free energy $\left(\Delta \Omega^{e x}\right)$ was calculated from the disjoining pressure (П) - total as well as individual contribution from confined water, cations and mica framework of opposite side. We also probed structural properties of water and ions by calculating several distributions. We found that analysis of various cation hydration scenarios (e.g., by surface oxygen, water, shared water) and three dimensional pair correlation function between ion and hydrating oxygen provides valuable informations. The key findings of this works are listed as below.

- Water adsorption in Na-, Li- and H-mica pores is qualitatively similar; however significant quantitative differences are observed, specially at smaller $d$. In these system, water molecules adsorbed even at dry conditions of mica having $d=4 \AA$, suggesting hygroscopic nature of these ions. Higher water density was expected in H-mica pores due smaller size of $\mathrm{H}^{+}$ions having higher hydration energy; however the counter-intuitive trend of $\rho_{L i}>\rho_{N a}>\rho_{b}>\rho_{H}$ was observed at smaller $d$. The reason was adsorption energy of water molecules in these systems which followed similar trend of density indicating that mica-framework also has significant contribution in energetics of the system.

- We found multiple hydration states of confined ions and water with variation in $d$. The $\mathrm{Na}^{+}$ion is adsorbed in the center of the pore for $d \leq 5$ forming IS complexes with adsorbed water. The equilibrium adsorption position of $\mathrm{Na}^{+}$ion shifts towards the surfaces with increase in $d$, splitting into two IS configurations at $d=7.5 \AA$, and remerging to form broad and distributed peak adjacent to each surface at $d \geq 9.5 \AA$. The $\mathrm{Li}^{+}$ions are mostly observed in IS state for all $d$, except for $d=6.5-7.5 \AA$, 
where significant fraction of $\mathrm{Li}^{+}$ions are adsorbed in the center of the pore forming OS complexes with adsorbed water. The $\mathrm{H}^{+}$ions are adsorbed in multiple layer inside the pore at all $d$. Only for $d \leq 6.5 \AA, \mathrm{H}^{+}$ions are present in IS state whereas for the rest of the pores, majority of cations are present in OS or diffusive states, away from the surface.

- In all three system, continuous evolution in water structure (preferential location and orientation) for $d \leq 11 \AA$ is observed due to adsorption density of water, hydration of cations, and, formation of water-water and water-surface hydrogen bonds.

- The swelling pressure $(\Pi)$ and free energy $\left(\Delta \Omega^{e x}\right)$ profiles show the oscillatory behavior with diminishing to zero for $d \geq 11 \AA$ in all three mica system. The location of optima in the $\Delta \Omega^{e x}$ is different suggesting that cations have significant role to play. A shift in location of global minima of $\Delta \Omega^{e x}$ towards the higher $d$ values and decrease in attractive $\Delta \Omega^{e x}$ is observed in the increasing order of hydration energy of $\mathrm{Na}^{+}, \mathrm{Li}^{+}$, and $\mathrm{H}^{+}$ions. In Na-mica system, the energy barrier for crystalline swelling from $d=6$ to $9 \AA$, is observed whereas the barrier is lower for crystalline $(d=9 \AA)$ to osmotic swelling $(d>12 \AA)$. For Li-mica, the energy barrier for crystalline to osmotic swelling is lesser compared to Na-mica system, whereas for H-mica the $\Delta \Omega^{e x}>0$ for all $d$ thus osmotic swelling is favoured.

- We found that cation hydration by surface atoms play a key role in deciding the swelling ability of clay. The $\mathrm{Na}^{+}$ion which has lesser hydration energy and larger size, they comfortably fit in the ditrigonal cavity of mica surface and simultaneously hydrate more number of surface oxygen atoms. On each side of mica frameworks, the strongly adsorbed $\mathrm{Na}^{+}$ions acts as anchors and hold the mica pore together at $d_{\min }=6 \AA$ by sharing of water in their hydration shell with the opposite side ion forming an electrostatically connected bridge of mica-Na-water-Na-mica.

- The $\mathrm{Li}^{+}$ions which has moderate hydration energy and average size, they hydrate 
surface oxygen atoms, albeit lesser number and sharing of hydration shell with nearby $\mathrm{Li}^{+}$ions is also minimum at $d_{m i n}=9 \AA$. Specifically the fraction of ions hydrating multiple surface oxygen atoms simultaneously is very less at $d_{\min }=9 \AA$. In between $d=7-9 \AA$, the cations prefer to detach from surface, adsorb in the center of the pore and maintain hydration shell with water molecules due to higher hydration energy. Only at extreme confinement of $d \leq 6 \AA$ the simultaneous hydration of more than one surface oxygen atom by $\mathrm{Li}^{+}$ion is observed.

- The tendency of cation detachment from mica surface is observed in the $\mathrm{H}^{+}$ions also which has smallest size and largest hydration energy amongst the cations studied. The surface hydration and water sharing, both, is minimum as the $\mathrm{H}^{+}$ions are mostly present in the center of the pore as diffusive ions, thus exerting a constant osmotic pressure on the mica frameworks, favouring swelling.

This detailed understanding of change in clay swelling behaviour due to differences in hydration properties of interstitial cations will provide aid in experimental analysis and design of better inhibiting materials.

\section{Acknowledgement}

AM acknowledge DST India (CRG/2020/004276) for financial support and both authors acknowledge IITB HPC facility (spacetime) for computational support.

\section{Supporting Information Available}

Supporting Information contains additional information on i) forcefield parameters, ii) structure of water adjacent to single M-mica surface, and iii) density of water and ions in various pores of Na-, Li- and H-mica systems. 


\section{References}

(1) Sena, M. M.; Morrow, C. P.; Kirkpatrick, R. J.; Krishnan, M. Supercritical Carbon Dioxide at Smectite Mineral-Water Interfaces: Molecular Dynamics and Adaptive Biasing Force Investigation of $\mathrm{CO}_{2} / \mathrm{H}_{2} \mathrm{O}$ Mixtures Nanoconfined in Na-Montmorillonite. Chem. Mater. 2015, 27, 6946.

(2) Hanczyc, M. M.; Fujikawa, S. M.; Szostak, J. W. Experimental Models of Primitive Cellular Compartments: Encapsulation, Growth, and Division. Science 2003, 302, 618.

(3) Carretero, M. Clay minerals and their beneficial effects upon human health. A review. Appl. Clay Sci. 2002, 21, 155.

(4) Greathouse, J. A.; Cygan, R. T.; Fredrich, J. T.; Jerauld, G. R. Molecular Dynamics Simulation of Diffusion and Electrical Conductivity in Montmorillonite Interlayers. $J$. Phys. Chem. C 2016, 120, 1640.

(5) Ma, Z.; Gamage, R. P.; Rathnaweera, T.; Kong, L. Review of application of molecular dynamic simulations in geological high-level radioactive waste disposal. Appl. Clay Sci. 2019, 168, 436 .

(6) Dixon, J. B. Roles of clay in soils. Appl. Clay Sci. 1991, 5, 489.

(7) Gartner, E.; Hirao, H. A review of alternative approaches to the reduction of $\mathrm{CO} 2$ emissions associated with the manufacture of the binder phase in concrete. Cem. Concr. Res. 2015, 78, 126.

(8) Murray, H. H. Traditional and new applications for kaolin, smectite, and palygorskite: A general overview. Appl. Clay Sci. 2000, 17, 207.

(9) Garrido-Ramirez, E.; Theng, B.; Mora, M. Clays and oxide minerals as catalysts and nanocatalysts in Fenton-like reactions- A review. Appl. Clay Sci. 2010, 47, 182. 
(10) Rotenberg, B.; Marry, V.; Vuilleumier, R.; Malikova, N.; Simon, C.; Turq, P. Water and ions in clays: Unraveling the interlayer/micropore exchange using molecular dynamics. Geochim. Cosmochim. Acta 2007, 71, 5089.

(11) Whittaker, M. L.; Lammers, L. N.; Carrero, S.; Gilbert, B.; Banfield, J. F. Ion exchange selectivity in clay is controlled by nanoscale chemical-mechanical coupling. Proc. Nat. Acad. Sci. 2019, 116, 22052.

(12) Planková, B.; Lísal, M. Molecular dynamics of aqueous salt solutions in clay nanopores under the thermodynamic conditions of hydraulic fracturing: Interplay between solution structure and molecular diffusion. Fluid Phase Equilibr. 2020, 505, 112355.

(13) Hofmann, U.; Endell, K.; Wilm, D. Kristallstruktur und Quellung von Montmorillonit. Z. Krist. 1933, 86, 340 .

(14) Norrish, K. THE SWELLING OF MONTMORILLONITE. Discuss. Faraday Soc. $\mathbf{1 9 5 4 ,} 18,120$.

(15) Christidis, G.; Blum, A.; Eberl, D. Influence of layer charge and charge distribution of smectites on the flow behaviour and swelling of bentonites. Appl. Clay Sci. 2006, 34, 125.

(16) Anderson, R.; Ratcliffe, I.; Greenwell, H.; Williams, P.; Cliffe, S.; Coveney, P. Clay swelling - A challenge in the oilfield. Earth-Sci. Rev. 2010, 98, 201.

(17) Mooney, R. W.; KEENAN, G.; WOOD, L. A. Adsorption of Water Vapor by Montmorillonite. II. Effect of Exchangeable Ions and Lattice Swelling as Measured by X-Ray Diffraction. J. Am. Chem. Soc. 1952, 74, 1371.

(18) Berend, I.; CASES, J.-M.; FRANCOIS, M.; URIOT, J.-P.; MICHOT, L.; MASION, A.; THOMAS, F. MECHANISM OF ADSORPTION AND DESORPTION OF WATER 
VAPOR BY HOMOIONIC MONTMORILLONITES: 2. THE $\mathrm{Li}^{+}, \mathrm{Na}^{+}, \mathrm{K}^{+}, \mathrm{Rb}^{+}$AND $\mathrm{Cs}^{+}$-EXCHANGED FORMS. Clays Clay Miner. 1995, 43, 324.

(19) TAMURA, K.; YAMADA, H.; NAKAZAWA, H. STEPWISE HYDRATION OF HIGHQUALITY SYNTHETIC SMECTITE WITH VARIOUS CATIONS. Clays Clay Miner. 2000, 48, 400 .

(20) Rongwei, S.; Tsukahara, T. Effect of Cations on Interlayer Water Dynamics in Cation-Exchanged Montmorillonites Studied by Nuclear Magnetic Resonance and Xray Diffraction Techniques. ACS Earth Space Chem. 2020, 4, 535.

(21) Posner, A. M.; Quirk, J. P. CHANGES IN BASAL SPACING OF MONTMORILLONITE IN ELECTROLYTE SOLUTIONS. J. Colloid. Sci. 1964, 19, 798.

(22) He, Y.; Ye, W.-M.; Chen, Y.-G.; Cui, Y.-J. Effects of K+ solutions on swelling behavior of compacted GMZ bentonite. Eng. Geol. 2019, 249, 241.

(23) Subramanian, N.; Whittaker, M. L.; Ophus, C.; Lammers, L. N. Structural Implications of Interfacial Hydrogen Bonding in Hydrated Wyoming-Montmorillonite Clay. J. Phys. Chem. C 2020, 124, 8697.

(24) MOOnEY, R. W.; KEEnAN, G.; WOOD, L. A. Adsorption of Water Vapor by Montmorillonite. I. Heat of Desorption and Application of BET Theory. J. Am. Chem. Soc. 1952, 74, 1367.

(25) Ferrage, E.; Lanson, B.; Michot, L. J.; Robert, J.-L. Hydration Properties and Interlayer Organization of Water and Ions in Synthetic Na-Smectite with Tetrahedral Layer Charge. Part 1. Results from X-ray Diffraction Profile Modeling. J. Phys. Chem. C $2010,114,4515$.

(26) Ferrage, E.; Sakharov, B. A.; Michot, L. J.; Delville, A.; Bauer, A.; Lanson, B.; Grangeon, S.; Frapper, G.; Jiménez-Ruiz, M.; Cuello, G. J. Hydration Properties and 
Interlayer Organization of Water and Ions in Synthetic Na-Smectite with Tetrahedral Layer Charge. Part 2. Toward a Precise Coupling between Molecular Simulations and Diffraction Data. J. Phys. Chem. C 2011, 115, 1867.

(27) Michot, L. J.; Ferrage, E.; Jiménez-Ruiz, M.; Boehm, M.; Delville, A. Anisotropic Features of Water and Ion Dynamics in Synthetic Na- and Ca-Smectites with Tetrahedral Layer Charge. A Combined Quasi-elastic Neutron-Scattering and Molecular Dynamics Simulations Study. J. Phys. Chem. C 2012, 116, 16619.

(28) Díaz-Pérez, A.; Cortés-Monroy, I.; Roegiers, J. The role of water/clay interaction in the shale characterization. J. Pet. Sci. Eng. 2007, 58, 83.

(29) Dazas, B.; Lanson, B.; Delville, A.; Robert, J.-L.; Komarneni, S.; Michot, L. J.; Ferrage, E. Influence of Tetrahedral Layer Charge on the Organization of Interlayer Water and Ions in Synthetic Na-Saturated Smectites. J. Phys. Chem. C 2015, 119, 4158.

(30) Martin-Jimenez, D.; Chacon, E.; Tarazona, P.; Garcia, R. Atomically resolved threedimensional structures of electrolyte aqueous solutions near a solid surface. Nat. Commun. 2016, 7, 12164.

(31) Ricci, M.; Spijker, P.; Voïtchovsky, K. Water-induced correlation between single ions imaged at the solid-liquid interface. Nat. Commun. 2014, 5, 4400.

(32) T. Li, J. G.; Szoszkiewicz, R.; Landman, U.; Riedo, E. Structured and viscous water in subnanometer gaps. Phys. Rev. B 2007, 75, 115415.

(33) Israelachvili, J. N.; Adams, G. E. Measurement of forces between two mica surfaces in aqueous electrolyte solutions in the range 0-100 nm. J. Chem. Soc., Faraday Trans. 1978, 74, 975-1001.

(34) Pashley, R. M. DLVO and hydration forces between mica surfaces in $\mathrm{Li}^{+}, \mathrm{Na}^{+}, \mathrm{K}^{+}$, 
and $\mathrm{Cs}^{+}$electrolyte solutions: A correlation of double-layer and hydration forces with surface cation exchange properties. J. Colloid Interface Sci. 1981, 83, 531.

(35) Pashley, R. M.; Israelachvili, J. N. Molecular Layering of Water in Thin Films between Mica Surfaces and Its Relation to Hydration Forces. J. Colloid Interface Sci. 1984, $101,511-523$.

(36) Raviv, U.; Klein, J. Fluidity of bound hydration layers. Science 2002, 297, 1540.

(37) Derjaguin, B.; Landau, L. Theory of the stability of strongly charged lyophobic sols and of the adhesion of strongly charged particles in solutions of electrolytes. Prog. Surf. Sci. 1993, 43, 30 .

(38) Verwey, E. J. W.; Overbeek, J. T. G. Theory of the Stability of Lyophobic Colloids; Dover, 1948.

(39) Israelachvili, J.; Wennerstrom, H. Role of hydration and water strucutre in biological and colloidal interactions. Nature 1996, 379, 219.

(40) van Lin, S. R.; Grotz, K. K.; Siretanu, I.; Schwierz, N.; Mugele, F. Ion-Specific and pH-Dependent Hydration of Mica-Electrolyte Interfaces. Langmuir 2019, 35, 5737.

(41) Diestler, D. J.; Schoen, M.; Curry, J. E.; Cushman, J. H. Thermodynamics of a fluid confined to a slit pore with structured walls. J. Chem. Phys. 1994, 100, 9140.

(42) Israelachvili, J. N. Measurement of the Viscosity of Liquids in Very Thin Films. J. Colloid Interface Sci. 1986, 110, 263.

(43) Raviv, U.; Laurat, P.; Klein, J. Fluidity of water confined to subnanometer films. Nature 2001, 413, 51.

(44) Zhu, Y.; Granick, S. Viscosity of Interfacial Water. Phys. Rev. Lett. 2001, 87, 096104. 
(45) Perkin, S.; Goldberg, R.; Chai, L.; Kampf, N.; Klein, J. Dynamic properties of confined hydration layers. Faraday Dis. 2009, 141, 399-413.

(46) Hsiao, Y.-W.; Hedström, M. Swelling Pressure in Systems with Na-Montmorillonite and Neutral Surfaces: A Molecular Dynamics Study. J. Phys. Chem. C 2017, 121, 26414.

(47) Young, D. A.; Smith, D. E. Simulations of Clay Mineral Swelling and Hydration: Dependence upon Interlayer Ion Size and Charge. J. Phys. Chem. B 2000, 104, 9163.

(48) Boek, E. S.; Coveney, P. V.; Skipper, N. T. Monte Carlo Molecular Modeling Studies of Hydrated Li-, Na- and K-Smectites: Understanding the Role of Potassium as a Clay Swelling Inhibitor. J. Am. Chem. Soc. 1995, 117, 12608-12617.

(49) Boek, E. S.; Coveney, P. V.; Skipper, N. T. Molecular Modeling of Clay Hydration: A Study of Hysteresis Loops in the Swelling Curves of Sodium Montmorillonites. Langmuir 1995, 11, 4629-4631.

(50) Karaborni, S.; Smit, B.; Heidug, W.; Urai, J.; van Oort, E. The Swelling of Clays: Molecular Simulations of the Hydration of Montmorillonite. Science 1996, 271, 11021104.

(51) Smith, D. E. Molecular Computer Simulations of the Swelling Properties and Interlayer Structure of Cesium Montmorillonite. Langmuir 1998, 14, 5959.

(52) Malani, A.; Ayappa, K. G.; Murad, S. Influence of Hydrophilic Surface Specificity on the Structural Properties of Confined Water. J. Phys. Chem. B 2009, 113, 13825.

(53) Seppala, A.; Puhakka, E.; Olin, M. Effect of layer charge on the crystalline swelling of $\mathrm{Na}+, \mathrm{K}+$ and $\mathrm{Ca} 2+$ montmorillonites: DFT and molecular dynamics studies. Clay Minerals 2016, 51, 197. 
(54) Zheng, Y.; Zaoui, A.; Shahrour, I. A theoretical study of swelling and shrinking of hydrated Wyoming montmorillonite. Appl. Clay Sci. 2011, 51, 177.

(55) Teich-McGoldrick, S. L.; Greathouse, J. A.; Jové-Colón, C. F.; Cygan, R. T. Swelling Properties of Montmorillonite and Beidellite Clay Minerals from Molecular Simulation: Comparison of Temperature, Interlayer Cation, and Charge Location Effects. J. Phys. Chem. C 2015, 119, 20880.

(56) Liu, X.-D.; Lu, X.-C. A Thermodynamic Understanding of Clay-Swelling Inhibition by Potassium Ions. Angew. Chem. Int. Ed. 2006, 45, 6300.

(57) Tambach, T. J.; Bolhuis, P. G.; Hensen, E. J. M.; Smit, B. Hysteresis in Clay Swelling Induced by Hydrogen Bonding: Accurate Prediction of Swelling States. Langmuir 2006, 22, 1223.

(58) Whitley, H. D.; Smith, D. E. Free energy, energy, and entropy of swelling in Cs-, Na-, and Sr-montmorillonite clays. J. Chem. Phys. 2004, 120, 5387.

(59) Smith, D. E.; Wang, Y.; Whitley, H. D. Molecular simulations of hydration and swelling in clay minerals. Fluid Phase Equilibr. 2004, 222-223, 189.

(60) Rahromostaqim, M.; Sahimi, M. Molecular Dynamics Simulation of Hydration and Swelling of Mixed-Layer Clays. J. Phys. Chem. C 2018, 122, 14631.

(61) Li, X.; Li, Q.; Yang, S.; Yang, G. Swelling of clay minerals: Dual characteristics of K+ ions and exploration of critical influencing factors. Phys. Chem. Chem. Phys. 2019, 21, 1963.

(62) Shroll, R. M.; Smith, D. E. Molecular dynamics simulations in the grand canonical ensemble: Application to clay mineral swelling. J. Chem. Phys. 1999, 111, 9025. 
(63) Shen, X.; Bourg, I. C. Molecular dynamics simulations of the colloidal interaction between smectite clay nanoparticles in liquid water. J. Colloid Interface Sci. 2021, 584, 610.

(64) Li, Y.-C.; Wei, S.-J.; Xu, N.; He, Y. Internal Forces within the Layered Structure of NaMontmorillonite Hydrates: Molecular Dynamics Simulation. J. Phys. Chem. C 2020, $124,25557$.

(65) Joung, I. S.; Cheatham III, T. E. Determination of Alkali and Halide Monovalent Ion Parameters for Use in Explicitly Solvated Biomolecular Simulations. J. Phys. Chem. B 2008, 112, 9020 .

(66) Marcus, Y. Thermodynamics of Solvation of Ions. J. Chem. Soc., Faraday Trans. 1991, $87,2995$.

(67) Debbarma, R.; Malani, A. Comparative Study of Water Adsorption on a $\mathrm{H}^{+}$and $\mathrm{K}^{+}$ Ion Exposed Mica Surface: Monte Carlo Simulation Study. Langmuir 2016, 32, 1034.

(68) Adapa, S.; Swamy, D. R.; Kancharla, S.; Pradhan, S.; Malani, A. Role of Mono- and Divalent Surface Cations on the Structure and Adsorption Behavior of Water on Mica Surface. Langmuir 2018, 34, 14472.

(69) Allen, M. P.; Tildesley, D. J. Computer Simulation of Liquids; Oxford University Press: New York, USA, 1987.

(70) Frenkel, D.; Smit, B. Understanding Molecular Simulation; Academic Press: San Diego, USA, 2002.

(71) Yeh, I. C.; Berkowitz, M. L. Ewald summation for systems with slab geometry. J. Chem. Phys. 1999, 111, 3155-3162.

(72) Adapa, S.; Malani, A. Role of Cation Size on Swelling Pressure and Free Energy of Mica Pores. J. Colloid Interface Sci. 2021, 599, 694. 
(73) Cygan, R. T.; Liang, J. J.; Kalinichev, A. G. Molecular Models of Hydroxide, Oxyhydroxide, and Clay Phases and the Development of a General Force Field. J. Phys. Chem. B 2004, 108, 1255-1266.

(74) Berendsen, H. J. C.; Grigera, J. R.; Straatsma, T. P. The missing term in effective pair potentials. J. Phys. Chem. 1987, 91, 6269.

(75) MacKerell Jr., A. D. et al. All-Atom Empirical Potential for Molecular Modeling and Dynamics Studies of Proteins. J. Phys. Chem. B 1998, 102, 3586.

(76) Ryckaert, J. P.; Ciccotti, G.; Berendsen, H. J. Numerical integration of the cartesian equations of motion of a system with constraints: Molecular dynamics of n-alkanes. J. Compt. Phys. 1977, 23, 327.

(77) Plimpton, S. Fast Parallel Algorithms for Short-Range Molecular Dynamics. J. Compt. Phys. 1995, $117,1$.

(78) Magda, J. J.; M.Tirrell,; Davis, H. T. Molecular dynamics of narrow, liquid filled pores. J. Chem. Phys. 1985, 83, 1888-1901.

(79) Gao, J.; Luedtke, W. D.; Landman, U. Origins of Solvation Forces in Confined Films. J. Phys. Chem. B 1997, 101, 4013-4023.

(80) Ash, S. G.; Everett, D. H.; Radke, C. Thermodynamics of the effects of adsorption on interparticle forces. J. Chem. Soc. 1973, 69, 1256.

(81) Evans, R.; Marini Bettolo Marconi, U. Phase equilibria and solvation forces for fluids confined between parallel walls. J. Chem. Phys. 1987, 86, 7138.

(82) Ghatak, C.; Ayappa, K. G. Solvation force, structure and thermodynamics of fluids confined in geometrically rough pores. J. Chem. Phys. 2004, 120, 9703.

(83) Bourg, I. C.; Lee, S. S.; Fenter, P.; Tournassat, C. Stern Layer Structure and Energetics at Mica-Water Interfaces. J. Phys. Chem. C 2017, 121, 9402. 
(84) Lee, S. S.; Fenter, P.; Nagy, K. L.; Sturchio, N. C. Monovalent ion adsorption at the muscovite (001)-solution interface: relationships among ion coverage and speciation, interfacial water structure, and substrate relaxation. Langmuir 2012, 28, 8637.

(85) Kobayashi, K.; Liang, Y.; Murata, S.; Matsuoka, T.; Takahashi, S.; Nishi, N.; Sakka, T. Ion Distribution and Hydration Structure in the Stern Layer on Muscovite Surface. Langmuir 2017, 33, 3892.

(86) Sakuma, H.; Kondo, T.; Nakao, H.; Shiraki, K.; Kawamura, K. Structure of Hydrated Sodium Ions and Water Molecules Adsorbed on the Mica/Water Interface. J. Phys. Chem. C 2011, 115, 15959.

(87) Schlegel, M. L.; Nagy, K. L.; Fenter, P.; Cheng, L.; Sturchio, N. C.; Jacobsen, S. D. Cation sorption on the muscovite (001) surface in chloride solutions using highresolution X-ray reflectivity. Geochim. Cosmochim. Acta 2006, 70, 3549.

(88) Adapa, S.; Malani, A. Role of hydration energy and co-ions association on monovalent and divalent cations adsorption at mica-aqueous interface. Sci. Rep. 2018, 8, 12198.

(89) Somers, S. A.; Davis, H. T. Microscopic dynamics of fluids confined between smooth and structured surfaces. J. Chem. Phys. 1992, 96, 5389.

(90) Somers, S. A.; McCormick, A. V.; Davis, H. T. Superselectivity and solvation forces of a two component fluid adsorbed in slit micropores. J. Chem. Phys. 1993, 99, 9890-9898.

(91) Davis, H. T. Statistical Mechanics of Phases, Interfaces and Thin Films; VCH Publishers Inc.: New York, USA, 1996.

(92) Klein, J.; Kumacheva, E. Confinement-induced phase transitions in simple liquids. Science 1995, 269, 816.

(93) Malani, A.; Ayappa, K. G. Confined fluids in a Janus pore: influence of surface asymmetry on structure and solvation forces. Mol. Sim. 2012, 38, 1114. 
(94) Ayappa, K. G.; Mishra, R. K. Freezing of fluids confined between mica surfaces. J. Phys. Chem. B 2007, 111, 14299-14310. 